\title{
Integrable discrete Schrödinger equations and a characterization of Prym varieties by a pair of quadrisecants
}

\author{
S.Grushevsky * $\quad$ I.Krichever ${ }^{\dagger}$
}

\begin{abstract}
We prove that Prym varieties are characterized geometrically by the existence of a symmetric pair of quadrisecant planes of the associated Kummer variety. We also show that Prym varieties are characterized by certain (new) theta-functional equations. For this purpose we construct and study a difference-differential analog of the NovikovVeselov hierarchy.
\end{abstract}

\section{Introduction}

An involution $\sigma: \Gamma \longmapsto \Gamma$ of a smooth algebraic curve $\Gamma$ induces an involution $\sigma^{*}: J(\Gamma) \longmapsto$ $J(\Gamma)$ of the Jacobian of the curve. The odd subspace under this involution, i.e. the set of $z \in J(\Gamma)$ such that $\sigma^{*}(z)=-z$, as the kernel of a homomorphism of abelian varieties, is the sum of a lower-dimensional abelian variety, called the Prym variety (the connected component of zero in the odd subspace), and a finite group. The Prym variety naturally has a polarization induced by the principal polarization on the Jacobian. However, this polarization is not principal, and the Prym variety admits a natural principal polarization if and only if $\sigma$ has at most two fixed points on $\Gamma$ - this is the case we will concentrate on.

The problem of characterizing the locus $\mathcal{P}_{g}$ of Pryms of dimension $g$ in the moduli space $\mathcal{A}_{g}$ of all principally polarized abelian varieties (ppav's) is well-known and has attracted a lot of interest over the years. In some sense the Prym varieties may be geometrically the easiest to understand ppavs beyond Jacobians, and one could hope that studying them would be a first step towards understanding the geometry of more general abelian varieties as well.

Recently, the Prym varieties for the case of an involution with two fixed points were characterized in [16] by one of the authors by the property of the theta function satisfying a

${ }^{*}$ Department of Mathematics, Princeton University, Princeton, NJ 08544, USA; e-mail: sam@math.princeton.edu. Research is supported in part by National Science Foundation under the grant DMS-05-55867.

${ }^{\dagger}$ Columbia University, New York, USA and Landau Institute for Theoretical Physics, Moscow, Russia; e-mail: krichev@math.columbia.edu. Research is supported in part by National Science Foundation under the grant DMS-04-05519. 
certain partial differential equation, coming from the theory of integrable Schrödinger equations. The main goal of this paper is to give a geometric (and equivalent theta-functional) characterization of Prym varieties corresponding to involutions with no fixed points (the Prym varieties of involutions with two fixed points arise as a degeneration of this case).

Let $B$ be an indecomposable complex symmetric matrix with positive definite imaginary part. It defines an indecomposable ppav $X:=\mathbb{C}^{g} / \Lambda$, where $\Lambda:=\mathbb{Z}^{g}+B \mathbb{Z}^{g} \subset \mathbb{C}^{g}$. The Riemann theta function is given by the formula

$$
\theta(B, z):=\sum_{m \in \mathbb{Z}^{g}} e^{2 \pi i(z, m)+\pi i(B m, m)}, \quad(z, m)=m_{1} z_{1}+\ldots+m_{g} z_{g}
$$

for $z \in \mathbb{C}^{g}$. The theta functions of the second order are defined by the formula

$$
\Theta[\varepsilon](B, z):=\sum_{m \in \mathbb{Z}^{g}} e^{2 \pi i(2 m+\varepsilon, z)+\pi i\left(2 m+\varepsilon, B\left(m+\frac{\varepsilon}{2}\right)\right)}
$$

for $\varepsilon \in(\mathbb{Z} / 2 \mathbb{Z})^{g}$. The Kummer variety $K(X)$ is then defined as the image of the Kummer map

$$
K: z \longmapsto\{\Theta[\varepsilon](z)\}_{\text {all } \varepsilon \in(\mathbb{Z} / 2 \mathbb{Z})^{g}} \in \mathbb{P}^{2^{g}-1} .
$$

A projective $(m-2)$-dimensional plane $\mathbb{P}^{m-2} \subset \mathbb{P}^{2^{g}-1}$ intersecting $K(X)$ in at least $m$ points is called an $m$-secant of the Kummer variety.

The Kummer images of Jacobians of curves were shown to admit many trisecant lines (see [9]). It was then shown by Gunning [11] that the existence of a one-dimensional family of trisecants in fact suffices to characterize Jacobians among all ppavs. Welters, inspired by the Gunning's theorem and the Novikov's conjecture proved later by Shiota [28], formulated in [29] the following conjecture: If $K(X)$ has a trisecant, and $X$ is indecomposable, then $X$ is a Jacobian, and this was recently proved by the second-named author in $([15,17])$.

Prym varieties possess generalizations of some properties that Jacobians possess. In [2] Beauville and Debarre, and in [10] Fay showed that the Kummer images of Prym varieties admit many quadrisecant planes. Similarly to the Jacobian case, it was then shown by Debarre in [5] that the existence of a one-dimensional family of quadrisecants characterizes Pryms. However, Beauville and Debarre in [2] showed that the existence of a single quadrisecant plane to the Kummer variety does not characterize Pryms.

In this paper we prove that the Prym varieties are characterized by the existence of a symmetric pair of quadrisecants of the corresponding Kummer variety - i.e. of two different 2-planes each intersecting the Kummer variety in 4 points, such that the points of secancy for the two planes are related in some precise way. We deduce this from a characterization by some theta-functional equations, and study the associated discrete Schrödinger equations along the way.

The structure of this work is as follows. In section 2 we prove that (the Kummer images of) Prym varieties have symmetric pairs of quadrisecants. This is done via an algebrogeometric construction of difference potential Schrödinger operators that play a crucial role in all our further considerations. Our construction is a discrete analog of the wellknown Novikov-Veselov algebro-geometric construction from [25] of a potential for the twodimensional Schrödinger operators. The latter is a reduction of a more general construction 
of finite-gap on one energy level Schrödinger operators in a magnetic field first proposed in [8] and based on the concept of the Baker-Akhiezer functions introduced in $[12,13]$.

The Baker-Akhiezer functions are uniquely defined by giving the data of an algebraic curve with fixed local coordinates in the neighborhoods of marked points, and by a divisor of its poles away from the marked points. These functions are not algebraic - they have essential singularities at marked point. To the authors' surprise in the case of unramified double covers the very same arguments that led Novikov and Veselov to a proof that certain constraints on the algebro-geometric spectral data of [8] are sufficient for potential reduction show that the poles divisor of the Baker-Akhiezer functions associated to unramified double covers of algebraic curves is of degree less than one would expect from the general theory. It turned out that this unexpected observation is equivalent to some well-known results of Mumford on Prym varieties [23].

In section 3 we introduce a discrete analog of the Novikov-Veselov hierarchy and study its properties. It is a set of difference-differential equations describing integrable deformations of potential difference Schrödinger operators. In section 4 of the paper, following the lines of works $[15,16,17]$ we construct a wave solution, and then in section 5 we finish the proof of our main result on the characterization of the Prym varieties of unramified covers. It is necessary to emphasize that, unlike the Jacobian case, the Prym variety remains compact under certain degenerations of the curve. No characterization of Prym varieties given in terms of the period matrix of the Prym differentials can single out the possibility of such degenerations.

Theorem 1.1 (Main theorem) An indecomposable principally polarized abelian variety $(X, \theta) \in \mathcal{A}_{g}$ is (in the closure of) the locus $\mathcal{P}_{g}$ of Prym varieties of unramified double covers if and only if there exist vectors $A, U, V, W \in \mathbb{C}^{g}$ representing distinct points in $X$, none of them points of order two, and constants $c_{1}, c_{2}, c_{3}, w_{1}, w_{2}, w_{3} \in \mathbb{C}$ such that one of the following equivalent conditions holds:

(A) The difference $2 D$ Schrödinger equation

$$
\psi_{n+1, m+1}-u_{n, m}\left(\psi_{n+1, m}-\psi_{n, m+1}\right)-\psi_{n, m}=0
$$

with

$$
u_{n, m}:=C_{n m} \frac{\theta\left((n+1) U+m V+\nu_{n m} W+Z\right) \theta\left(n U+(m+1) V+\nu_{n m} W+Z\right)}{\theta\left((n+1) U+(m+1) V+\left(1-\nu_{n m}\right) W+Z\right) \theta\left(n U+m V+\left(1-\nu_{n m}\right) W+Z\right)},
$$

where

$$
2 \nu_{n m}:=1+(-1)^{n+m+1}, \quad C_{n m}:=c_{3}\left(c_{2}^{2 n+1} c_{1}^{2 m+1}\right)^{1-2 \nu_{n m}}
$$

and

$$
\psi_{n, m}:=\frac{\theta\left(A+n U+m V+\nu_{n m} W+Z\right)}{\theta\left(n U+m V+\left(1-\nu_{n m}\right) W+Z\right)} w_{1}^{n} w_{2}^{m} w_{3}^{\nu_{n m}}\left(c_{1}^{m} c_{2}^{n}\right)^{1-2 \nu_{n m}} .
$$

is satisfied for all $Z \in X$. 
(B) The following identity holds:

$$
\begin{aligned}
& w_{1} w_{2}\left(c_{1} c_{2}\right)^{ \pm 1} \widetilde{K}\left(\frac{A+U+V \mp W}{2}\right)-w_{1} c_{3}\left(w_{3} c_{1}\right)^{ \pm 1} \widetilde{K}\left(\frac{A+U-V \pm W}{2}\right) \\
+ & w_{2} c_{3}\left(w_{3} c_{2}\right)^{ \pm 1} \widetilde{K}\left(\frac{A+V-U \pm W}{2}\right)-\widetilde{K}\left(\frac{A-U-V \mp W}{2}\right)=0
\end{aligned}
$$

where $\widetilde{K}: \mathbb{C}^{g} \rightarrow \mathbb{C}^{2^{g}}$ is the lifting of the Kummer map to the universal cover.

$(C)$ The two equations (one for the top choice of signs everywhere, and one - for the bottom)

$$
\begin{array}{r}
c_{1}^{\mp 2} c_{3}^{2} \theta(Z+U-V) \theta(Z-U \pm W) \theta(Z+V \pm W) \\
+c_{2}^{\mp 2} c_{3}^{2} \theta(Z-U+V) \theta(Z+U \pm W) \theta(Z-V \pm W) \\
=c_{1}^{\mp 2} c_{2}^{\mp 2} \theta(Z-U-V) \theta(Z+U \pm W) \theta(Z+V \pm W) \\
+\theta(Z+U+V) \theta(Z-U \pm W) \theta(Z-V \pm W)
\end{array}
$$

are valid on the theta divisor $\{Z \in X: \theta(Z)=0\}$.

The purely geometric statement of part $(B)$ of this result is as follows.

Corollary 1.2 (Geometric characterization of Pryms) Given $(X, \theta) \in \mathcal{A}_{g}$, if there exist distinct points $p_{1}, p_{2}, p_{3}, p_{4} \in X$, none of them points of order two, such that the Kummer images of the eight points $p_{1} \pm p_{2} \pm p_{3} \pm p_{4}$ lie on two quadrisecants (the four points with the same parity of the number of plus signs forming each quadruple), then $(X, \theta)$ lies in the closure of the locus of Prym varieties of unramified double covers.

Proof. Indeed, statement $(B)$ gives the two linear dependencies for the Kummer images of the two quadruples of point. The 6 coefficients of linear dependence appearing in these two equations depend on 6 parameters $c_{i}, w_{i}$ and are independent (since all $c_{i}, w_{i}$ can be recovered from the 6 coefficients); thus $(B)$ says that any ppav admitting a symmetric pair of quadrisecants is a Prym.

The equivalence of $(A)$ and $(B)$ is a direct corollary of the addition formula for the theta function. The "only if" part of $(A)$ is what we prove in section 2 . The statement $(C)$ is actually what we use for the proof of the "if" part of the theorem. The characterization of Pryms by $(C)$ is stronger than the characterization by $(A)$. The implication $(A) \Rightarrow(C)$ does not require the explicit theta-functional formula for $\psi$. It is enough to require only that equation (1.1) with $u$ as in (1.2) has local meromorphic solutions which are holomorphic outside the divisor $\theta(U n+V m+Z)=0$ (see lemma 4.1).

It would be interesting to try to apply our geometric characterization of Pryms to studying other aspects of Prym geometry and of the geometry of the Prym locus, including the 
Torelli problem for Pryms, higher-dimensional secancy conditions, representability of homology classes in Pryms, etc. It is also tempting to ask whether a similar characterization of Prym-Tyurin varieties of higher order may be obtained, or whether one could use secancy conditions to geometrically stratify the moduli space of ppavs. We hope to pursue these questions in the future.

\section{Potential reduction of the algebro-geometric $2 D$ dif- ference Schrödinger operators}

To begin with let us recall a construction of algebro-geometric difference Schrödinger operators proposed in [18] (see details in [21]).

General notations, Baker-Akhiezer functions.

Let $\Gamma$ be a smooth algebraic curve of genus $\hat{g}$. Fix four points $P_{1}^{ \pm}, P_{2}^{ \pm} \in \Gamma$, and let $\hat{D}=\gamma_{1}+\cdots+\gamma_{\widehat{g}}$ be a generic effective divisor on $\Gamma$ of degree $\widehat{g}$. We denote by $B$ the period matrix of the curve $\Gamma$ (the integrals of a basis of the space of abelian differentials on $\Gamma$ over the $b$-cycles, once the integrals over the $a$-cycles are normalized), by $J(\Gamma)=\mathbb{C}^{\widehat{g}} / \mathbb{Z}^{\widehat{g}}+B \mathbb{Z}^{\widehat{g}}$ - the Jacobian variety of $\Gamma$, and by $\widehat{A}: \Gamma \hookrightarrow J(\Gamma)$ the Abel-Jacobi embedding of the curve into its Jacobian. We further denote by

$$
\widehat{\theta}(z):=\theta(B, z),
$$

the Riemann theta function of the variable $z \in \mathbb{C}^{\widehat{g}}$.

By the Riemann-Roch theorem one computes $h^{0}\left(\hat{D}+n\left(P_{1}^{+}-P_{1}^{-}\right)+m\left(P_{2}^{+}-P_{2}^{-}\right)\right)=1$, for any $n, m \in \mathbb{Z}$, and for $\hat{D}$ generic. We denote by $\widehat{\psi}_{n, m}(P), P \in \Gamma$ the unique section of this bundle. This means that $\widehat{\psi}_{n, m}$ is the unique up to a constant factor meromorphic function such that (away from the marked points $P_{i}^{ \pm}$) it has poles only at $\gamma_{s}$, of multiplicity not greater than the multiplicity of $\gamma_{s}$ in $\widehat{D}$, while at the points $P_{1}^{+}, P_{2}^{+}$(resp. $P_{1}^{-}, P_{2}^{-}$) the function $\widehat{\psi}_{n, m}$ has poles (resp. zeros) of orders $n$ and $m$.

If we fix local coordinates $k^{-1}$ in the neighborhoods of marked points (it is customary in the subject to think of marked points as punctures, and thus it is common to use coordinates such that $k$ at the marked point is infinite rather than zero), then the Laurent series for $\psi_{n, m}(P)$, for $P \in \Gamma$ near a marked point, has the form

$$
\begin{aligned}
& \widehat{\psi}_{n, m}=k^{ \pm n}\left(\sum_{s=0}^{\infty} \xi_{s}^{ \pm}(n, m) k^{-s}\right), k=k(P), P \rightarrow P_{1}^{ \pm}, \\
& \widehat{\psi}_{n, m}=k^{ \pm m}\left(\sum_{s=0}^{\infty} \chi_{s}^{ \pm}(n, m) k^{-s}\right), \quad k=k(P), P \rightarrow P_{2}^{ \pm} .
\end{aligned}
$$

Any meromorphic function on a Riemann surface can be expressed in terms of the theta functions, but it is easier to write an expression for $\widehat{\psi}_{n, m}$ using both the theta functions and the differentials of the third kind. Indeed, for $i=1,2$ let $d \widehat{\Omega}^{i} \in H^{0}\left(K_{\Gamma}+P_{i}^{+}+P_{i}^{-}\right)$be the 
differential of the third kind, normalized to have residues $\mp 1$ at $P_{i}^{ \pm}$and with zero integrals over all the $a$-cycles, and let $\widehat{\Omega}^{i}$ be the corresponding abelian integral, i.e. the function on the Riemann surface obtained by integrating $d \widehat{\Omega}^{i}$ from some fixed starting point to the variable point. Then we have the following expression

$$
\widehat{\psi}_{n, m}(P)=r_{n m} \frac{\widehat{\theta}(\widehat{A}(P)+n \widehat{U}+m \widehat{V}+\widehat{Z})}{\widehat{\theta}(\widehat{A}(P)+\widehat{Z})} e^{n \widehat{\Omega}_{1}(P)+m \widehat{\Omega}_{2}(P)},
$$

where $r_{n m}$ is some constant, $\widehat{U}=\widehat{A}\left(P_{1}^{-}\right)-\widehat{A}\left(P_{1}^{+}\right), \quad \widehat{V}=\widehat{A}\left(P_{2}^{-}\right)-\widehat{A}\left(P_{2}^{+}\right)$, and

$$
\widehat{Z}=-\sum_{s} \widehat{A}\left(\gamma_{s}\right)+\widehat{\kappa}
$$

where $\widehat{\kappa}$ is the vector of Riemann constants. Indeed, to prove that such an expression for $\widehat{\psi}_{n, m}$ is valid, one only needs to verify that both sides have the same zeros and poles, which is clear by construction.

Notation. From now on it will be useful to think of $n$ and $m$ as discrete variables, which are shifted by the shift operators that we denote $T_{1}: n \mapsto n+1$ and $T_{2}: m \mapsto m+1$ respectively. To emphasize the difference between the operator and its action, for a function $f=f(n, m)$ we will write $\mathbf{t}_{\mu} f:=T_{\mu} \circ f$, so that for example $T_{1}(f \cdot g)=\mathbf{t}_{1} f \cdot \mathbf{t}_{1} g$. We will also denote $H:=T_{1} T_{2}-u\left(T_{1}-T_{2}\right)-1$ the difference operator that is very important for what follows.

Theorem 2.1 ([18]) The Baker-Akhiezer function $\widehat{\psi}_{n, m}$ given by formula (2.3) satisfies the following difference equation

$$
\widehat{\psi}_{n+1, m+1}-a_{n, m} \widehat{\psi}_{n+1, m}-b_{n, m} \widehat{\psi}_{n, m+1}+c_{n, m} \widehat{\psi}_{n, m}=0,
$$

where we let

$$
\begin{gathered}
a_{n, m}:=\frac{\xi_{0}^{+}(n+1, m+1)}{\xi_{0}^{+}(n+1, m)}, \quad b_{n, m}:=\frac{\chi_{0}^{+}(n+1, m+1)}{\chi_{0}^{+}(n, m+1)}, \\
c_{n, m}:=b_{n, m} \frac{\xi^{-}(n, m+1)}{\xi_{0}^{-}(n, m)}=\frac{\xi^{-}(n, m+1) \chi_{0}^{+}(n+1, m+1)}{\xi_{0}^{-}(n, m) \chi_{0}^{+}(n, m+1)} .
\end{gathered}
$$

Explicit $\theta$-functional formulae for the coefficients follow from equation (2.3) which implies

$$
\begin{aligned}
& \xi_{0}^{ \pm}=r_{n m} \frac{\widehat{\theta}\left(\widehat{A}\left(P_{1}^{ \pm}\right)+n \widehat{U}+m \widehat{V}+\widehat{Z}\right)}{\widehat{\theta}\left(\widehat{A}\left(P_{1}^{ \pm}\right)+\widehat{Z}\right)} e^{n \alpha_{1}^{ \pm}+m \alpha_{2}^{ \pm}} \\
& \chi_{0}^{ \pm}=r_{n m} \frac{\widehat{\theta}\left(\widehat{A}\left(P_{2}^{ \pm}\right)+n \widehat{U}+m \widehat{V}+\widehat{Z}\right)}{\widehat{\theta}\left(\widehat{A}\left(P_{2}^{ \pm}\right)+\widehat{Z}\right)} e^{n \beta_{1}^{ \pm}+m \beta_{2}^{ \pm}}
\end{aligned}
$$

The constants $\alpha_{i}^{ \pm}, \beta_{i}^{ \pm}$are defined by the formulae:

$$
\begin{aligned}
& \alpha_{2}^{ \pm}=\Omega_{2}\left(P_{1}^{ \pm}\right) ; \quad \Omega_{1}= \pm \ln k+\alpha_{1}^{ \pm}+O\left(k^{-1}\right), \quad P \rightarrow P_{1}^{ \pm}, \\
& \beta_{1}^{ \pm}=\Omega_{1}\left(P_{2}^{ \pm}\right) ; \quad \Omega_{2}= \pm \ln k+\beta_{2}^{ \pm}+O\left(k^{-1}\right), \quad P \rightarrow P_{2}^{ \pm} .
\end{aligned}
$$




\section{Setup for the Prym construction}

We now assume that the curve $\Gamma$ is an algebraic curve endowed with an involution $\sigma$ without fixed points; then $\Gamma$ is a unramified double cover $\Gamma \longmapsto \Gamma_{0}$, where $\Gamma_{0}=\Gamma / \sigma$. If $\Gamma$ is of genus $\widehat{g}=2 g+1$, then by Riemann-Hurwitz the genus of $\Gamma_{0}$ is $g+1$. On $\Gamma$ one can choose a basis of cycles $a_{i}, b_{i}$ with the canonical matrix of intersections $a_{i} \cdot a_{j}=b_{i} \cdot b_{j}=0, a_{i} \cdot b_{j}=$ $\delta_{i j}, \quad 0 \leq i, j \leq 2 g$, such that under the involution $\sigma$ we have $\sigma\left(a_{0}\right)=a_{0}, \sigma\left(b_{0}\right)=b_{0}, \sigma\left(a_{j}\right)=$ $a_{g+j}, \sigma\left(b_{j}\right)=b_{g+j}, 1 \leq j \leq g$. If $d \omega_{i}$ are normalized holomorphic differentials on $\Gamma$ dual to this choice of $a$-cycles, then the differentials $d u_{j}=d \omega_{j}-d \omega_{g+j}$, for $j=1 \ldots g$ are odd, i.e. satisfy $\sigma^{*}\left(d u_{k}\right)=-d u_{k}$, and we call them the normalized holomorphic Prym differentials. The matrix of their $b$-periods

$$
\Pi_{k j}=\oint_{b_{k}} d u_{j}, \quad 1 \leq k, j \leq g,
$$

is symmetric, has positive definite imaginary part, and defines the Prym variety

$$
\mathcal{P}(\Gamma):=\mathbb{C}^{g} / \mathbb{Z}^{g}+\Pi \mathbb{Z}^{g}
$$

and the corresponding Prym theta function

$$
\theta(z):=\theta(\Pi, z),
$$

for $z \in \mathbb{C}^{g}$. We assume that the marked points $P_{1}^{ \pm}, P_{2}^{ \pm}$on $\Gamma$ are permuted by the involution, i.e. $P_{i}^{+}=\sigma\left(P_{i}^{-}\right)$. For further use let us fix in addition a third pair of points $P_{3}^{ \pm}$, such that also $P_{3}^{-}=\sigma\left(\mathcal{P}_{3}^{+}\right)$.

The Abel-Jacobi map $\Gamma \hookrightarrow J(\Gamma)$ induces the Abel-Prym map $A: \Gamma \longmapsto \mathcal{P}(\Gamma)$. There is a choice of the base point involved in defining the Abel-Jacobi map, and thus in the Abel-Prym map; let us choose this base point (such a choice is unique up to a point of order two in $\mathcal{P}(\Gamma))$ in such a way that

$$
A(P)=-A(\sigma(P)) .
$$

Admissible divisors. An effective divisor on $\Gamma$ of degree $\hat{g}-1=2 g, D=\gamma_{1}+\ldots \gamma_{2 g}$, is called admissible if it satisfies

$$
[D]+[\sigma(D)]=K_{\Gamma} \in J(\Gamma)
$$

(where $K_{\Gamma}$ is the canonical class of $\Gamma$ ), and if moreover $H^{0}(D+\sigma(D))$ is generated by an even holomorphic differential $d \Omega$, i.e. that

$$
d \Omega\left(\gamma_{s}\right)=d \Omega\left(\sigma\left(\gamma_{s}\right)\right)=0, \quad d \Omega=\sigma(d \Omega) .
$$

Algebraically, what we are saying is the following. The divisors $D$ satisfying (2.14) are the preimage of the point $K_{\Gamma}$ under the map $1+\sigma$, and thus are a translate of the subgroup $\operatorname{Ker}(1+\sigma) \subset J(\Gamma)$ by some vector. As shown by Mumford [22], this kernel has two components - one of them being the Prym, and the other being the translate of the Prym variety by the point of order two corresponding to the cover $\Gamma \rightarrow \Gamma_{0}$ as an element in 
$\pi_{1}\left(\Gamma_{0}\right)$. The existence of an even differential as above picks out one of the two components, and the other one is obtained by adding $A-\sigma(A)$ to the divisor of such a differential, for some $A$. In Mumford's notations the component we pick is in fact $P^{-}$(when we choose the base point according to (2.13) to identify $\mathrm{Pic}^{0}$ and $\mathrm{Pic}^{\widehat{g}-1}$ ), but throughout this paper we will have to deal with both components, using some point (which will be called $P_{3}^{+}$) and the corresponding shift by $P_{3}^{+}-P_{3}^{-}$to pass from one component to the other. We will prove the following statement.

Proposition 2.2 For a generic vector $Z$ the zero-divisor $D$ of the function $\theta(A(P)+Z)$ on $\Gamma$ is of degree $2 g$ and satisfies the constraints (2.14) and (2.15), i.e. is admissible.

Remark. We have been unable to find a proof of this statement in the literature. However, both Elham Izadi and Roy Smith have independently supplied us with simple proofs of this result, based on Mumford's description and results on Prym varieties. The reason we have chosen to still give the longer analytic proof below is because we need some of the intermediate results later on, and also to give an independent analytic proof of some of Mumford's results.

Note that the function $\theta(A(P)+Z)$ is multi-valued on $\Gamma$, but its zero-divisor is welldefined. The arguments identical to that in the standard proof of the inversion formula (2.4) show that the zero divisor $D(Z):=\theta(A(P)+Z)$ is of degree $\hat{g}-1=2 g$.

Lemma 2.3 For a generic $D=D(Z)$ and for each set of integers $(n, m, r)$ such that

$$
n+m+r=0 \bmod 2
$$

the space

$$
H^{0}\left(D+n\left(P_{1}^{+}-P_{1}^{-}\right)+m\left(P_{2}^{+}-P_{2}^{-}\right)+r\left(P_{3}^{+}-P_{3}^{-}\right)\right)
$$

is one-dimensional. A basis element of this space is given by

$$
\psi_{n, m, r}(P):=h_{n, m, r} \frac{\theta(A(P)+n U+m V+r W+Z)}{\theta(A(P)+Z)} e^{n \Omega_{1}(P)+m \Omega_{2}(P)+r \Omega_{3}(P)},
$$

where $\Omega_{j}$ is the abelian integral corresponding to a differential $d \Omega_{i}$ of the third kind, odd under the involution $\sigma$, and with residues $\mp 1$ at $P_{j}^{ \pm}$(i.e. $d \Omega_{i}=-\sigma\left(d \Omega_{j}\right)$ ), satisfying the normalization condition

$$
\oint_{a_{k}} d \Omega_{j}=\pi i l_{k}, \quad l_{k} \in \mathbb{Z}, \quad k=0, \ldots, 2 g,
$$

and $U, V, W$ are the vectors of b-periods of these differentials, i.e.

$$
2 \pi i U_{k}=\oint_{b_{k}} d \Omega_{1}, 2 \pi i V_{k}=\oint_{b_{k}} d \Omega_{2}, 2 \pi i W_{k}=\oint_{b_{k}} d \Omega_{3} .
$$


Proof. It is easy to check that the right hand side of (2.17) is a single valued function on $\Gamma$ having all the desired properties, and thus it gives a section of the desired bundle. Note that the constraint (2.16) is required due to (2.18), and the uniqueness of $\psi$ up to a constant factor, i.e. the one-dimensionality of the $H^{0}$ above, is a direct corollary of the Riemann-Roch theorem.

For further use let us note that bilinear Riemann identities imply

$$
2 U=A\left(P_{1}^{-}\right)-A\left(P_{1}^{+}\right), \quad 2 V=A\left(P_{2}^{-}\right)-A\left(P_{2}^{+}\right), \quad 2 W=A\left(P_{3}^{-}\right)-A\left(P_{3}^{+}\right) .
$$

Let us compare the definition of $\widehat{\psi}_{n, m}$ defined for any curve $\Gamma$, with that of $\psi_{n, m, r}$, which is only defined for a curve with an involution satisfying a number of conditions. To make such a comparison, consider the divisor $\widehat{D}=D+P_{3}^{+}$of degree $\hat{g}=2 g+1$, and let $\widehat{\psi}_{n, m}$ be the corresponding Baker-Akhiezer function.

Corollary 2.4 For the Baker-Akhiezer function $\widehat{\psi}_{n m}$ corresponding to the divisor $\widehat{D}=D+$ $P_{3}^{+}$we have

$$
\widehat{\psi}_{n m}=\psi_{n, m, \nu}
$$

where $\nu=\nu_{n m}$ is defined in (1.3), i.e. is 0 or 1 so that $n+m+\nu$ is even.

Corollary 2.5 If $n+m$ is even, then by formulae (2.3,2.17)

$$
\begin{aligned}
& \frac{\widehat{\theta}(\widehat{A}(P)+n \widehat{U}+m \widehat{V}+\widehat{Z}) \widehat{\theta}\left(\widehat{A}\left(P_{0}\right)+\widehat{Z}\right)}{\widehat{\theta}(\widehat{A}(P)+\widehat{Z}) \widehat{\theta}\left(\widehat{A}\left(P_{0}\right)+n \widehat{U}+m \widehat{V}+\widehat{Z}\right)}= \\
& \frac{\theta(A(P)+n U+m V+Z) \theta\left(A\left(P_{0}\right)+Z\right)}{\theta(A(P)+Z) \theta\left(A\left(P_{0}\right)+n U+m V+Z\right)} e^{n r_{1}+m r_{2}},
\end{aligned}
$$

where $r_{i}=\int_{P_{0}}^{P}\left(d \widehat{\Omega}_{i}-d \Omega_{i}\right)$, and we recall that $\widehat{Z}=\widehat{A}(\widehat{D})+\widehat{\kappa}$, and $Z$ is its image.

Remark 2.6 This equality, valid for any pair of points $P, P_{0}$ is a non-trivial identity between theta functions. The authors' attempts to derive it directly from the Schottky-Jung relations have failed so far.

Notation. For brevity throughout the rest of the paper we use the notation: $\psi_{n, m}:=$ $\psi_{n, m, \nu_{n m}}$.

Lemma 2.7 The Baker-Akhiezer function $\psi_{n, m}$ given by

$$
\psi_{n, m}=\frac{\theta\left(A(P)+U n+V m+\nu_{n m} W+Z\right)}{\theta\left(U n+V m+\left(1-\nu_{n m}\right) W+Z\right) \theta(A(P)+Z)} \cdot \frac{e^{n \Omega_{1}(P)+m \Omega_{2}(P)+\nu_{n m} \Omega_{3}(P)}}{e^{\left(2 \nu_{n m}-1\right)\left(n \Omega_{1}\left(P_{3}^{+}\right)+m \Omega_{2}\left(P_{3}^{+}\right)\right)}},
$$


satisfies the equation (1.1), i.e.

$$
\psi_{n+1, m+1}-u_{n, m}\left(\psi_{n+1, m}-\psi_{n, m+1}\right)-\psi_{n, m}=0,
$$

with $u_{n, m}$ as in (1.2,1.3), where

$$
c_{1}=e^{\Omega_{2}\left(P_{3}^{+}\right)}, \quad c_{2}=e^{\Omega_{1}\left(P_{3}^{+}\right)}, \quad c_{3}=e^{\Omega_{1}\left(P_{2}^{+}\right)}
$$

Proof. Note that the first and the last factors in the denominator of (2.23) correspond to a special choice of the normalization constants $h_{n, m, \nu}$ in (2.17):

$$
\begin{aligned}
\psi_{n m}\left(P_{3}^{-}\right) & =(\theta(Z+W))^{-1}, \quad \nu_{n m}=0, \\
\left.\psi_{n m} e^{-\Omega_{3}}\right|_{P=P_{3}^{+}} & =(\theta(Z-W))^{-1}, \quad \nu_{n m}=1 .
\end{aligned}
$$

This normalization implies that for even $n+m$ the difference $\left(\psi_{n+1, m+1}-\psi_{n, m}\right)$ equals zero at $P_{3}^{-}$. At the same time as a corollary of the normalization we get that $\left(\psi_{n+1, m}-\psi_{n, m+1}\right)$ has no pole at $P_{3}^{+}$. Hence, these two differences have the same analytical properties on $\Gamma$ and thus are proportional to each other (the relevant $H^{0}$ is one-dimensional by Riemann-Roch). The coefficient of proportionality $u_{n m}$ can be found by comparing the singularities of the two functions at $P_{1}^{+}$.

The second factor in the denominator of the formula (2.23) does not affect equation (1.1). Hence, the lemma proves the "only if" part of the statement $(A)$ of the main theorem for the case of smooth curves. It remains valid under degenerations to singular curves which are smooth outside of fixed points $Q_{k}$ which are simple double points, i.e. to the curves of type $\left\{\Gamma, \sigma, Q_{k}\right\}$.

Remark 2.8 Equation (1.1) as a special reduction of (2.5) was introduced in [7]. It was shown that equation (2.5) implies a five-term equation

$$
\psi_{n+1, m+1}-\tilde{a}_{n m} \psi_{n+1, m-1}-\tilde{b}_{n, m} \psi_{n-1, m+1}+\tilde{c}_{n m} \psi_{n-1, m-1}=\tilde{d}_{n, m} \psi_{n, m}
$$

if and only if it is of the form (1.1). A reduction of the algebro-geometric construction proposed in [18] in the case of algebraic curves with involution having two fixed points was found. It was shown that the corresponding Baker-Akhiezer functions do satisfy an equation of the form (1.1). Explicit formulae for the coefficients of the equations in terms of Riemann theta-functions were obtained. The fact that the Baker-Akhiezer functions and the coefficients of the equations can be expressed in terms of Prym theta-functions is new.

We are now ready to complete the proof of proposition (2.2). Let $\psi_{n, m}$ be the BakerAkhiezer function given by (2.23). According to Lemma 2.3 it satisfies equation (1.1). The differential $d \psi_{n, m}$ is also a solution of the same equation, and thus we get, using the shift operator notation,

$$
\left(T_{1}-1\right)\left(\psi_{n, m}^{\sigma} d \psi_{n, m+1}-\psi_{n, m+1}^{\sigma} d \psi_{n, m}\right)=\left(T_{2}-1\right)\left(\psi_{n, m}^{\sigma} d \psi_{n+1, m}-\psi_{n+1, m}^{\sigma} d \psi_{n, m}\right)
$$


For a generic set of algebro-geometrical spectral data the products $\psi_{n, m}^{\sigma} \psi_{n, m+1}$ and $\psi_{n, m}^{\sigma} \psi_{n+1, m}$ are quasi-periodic functions of the variables $n$ and $m$. The data for which they are periodic are characterized as follows.

Let $d p_{j}, i=1,2$ be the third kind abelian differentials with residues $\mp 1$ at the punctures $P_{j}^{ \pm}$, respectively, and normalized by the condition that all of their periods are purely imaginary,

$$
\Re \oint_{c} d p_{j}=0, \quad \forall c \in H^{1}(\Gamma, Z) .
$$

Non-degeneracy of the imaginary part of the period matrix of holomorphic differential implies that such $d p_{j}$ exists and is unique. If the periods of $d p_{j}$ are of the form

$$
\oint_{c} d p_{j}=\frac{\pi i n_{c}^{j}}{N_{j}}, n_{c}^{j} \in \mathbb{Z},
$$

then the function $\mu_{j}(Q)=e^{N_{j} \int^{Q} d p_{j}}$ is single-valued on $\Gamma$, has pole of order $N_{j}$ at $P_{j}^{+}$and zero of order $N_{j}$ at $P_{j}^{-}$. From the uniqueness of the Baker-Akhiezer function it then follows that

$$
\begin{aligned}
& \psi_{n+2 N_{1}, m}=\frac{\mu_{1}}{\mu_{1}\left(P_{3}^{-}\right)} \psi_{n, m}, \quad \psi_{n, m+2 N_{2}}=\frac{\mu_{2}}{\mu_{2}\left(P_{3}^{-}\right)} \psi_{n, m} \quad, \quad \nu=0 \\
& \psi_{n+2 N_{1}, m}=\frac{\mu_{1}}{\mu_{1}\left(P_{3}^{+}\right)} \psi_{n, m}, \quad \psi_{n, m+2 N_{2}}=\frac{\mu_{2}}{\mu_{2}\left(P_{3}^{+}\right)} \psi_{n, m} \quad, \quad \nu=1
\end{aligned}
$$

These imply

$$
\psi_{n+2 N_{1}, m}^{\sigma} d \psi_{n+1+2 N_{1}, m}=\psi_{n, m}^{\sigma} d \psi_{n+1, m}+\left(\psi_{n, m}^{\sigma} \psi_{n+1, m}\right) d p_{1}
$$

and similar monodromy properties for the other terms in (2.27). In this case the averaging of equation (2.27) in the variables $n, m$ gives the equation

$$
\left.\left\langle\psi^{\sigma} \mathbf{t}_{2} \psi-\mathbf{t}_{2} \psi^{\sigma}\right) \psi\right\rangle_{2} d p_{1}=\left\langle\psi^{\sigma}\left(\mathbf{t}_{1} \psi\right)-\left(\mathbf{t}_{1} \psi^{\sigma}\right) \psi\right\rangle_{1} d p_{2} .
$$

Here $\langle\cdot\rangle_{1}$ stands for the mean value in $n$ and $\langle\cdot\rangle_{2}$ stands for the mean value in $m$. For a generic curve differentials $d p_{j}$ have no common zeros. Hence, for such curves the differential

$$
d \Omega=\frac{d p_{1}}{\left\langle\psi^{\sigma} \mathbf{t}_{1} \psi-\mathbf{t}_{1} \psi^{\sigma} \psi\right\rangle_{1}}=\frac{d p_{2}}{\left\langle\psi^{\sigma} \mathbf{t}_{2} \psi-\mathbf{t}_{2} \psi^{\sigma} \psi\right\rangle_{2}}
$$

is holomorphic on $\Gamma$. It has zeros at the poles of $\psi$ and $\psi^{\sigma}$. The curves for which (2.29) holds for some $N_{j}$ are dense in the moduli space of all smooth genus $g$ curves. That proves that equation (2.33) holds for any curve. Proposition (2.2) is proven.

Remark 2.9 We have thus proven that for any Prym variety part (A) of the main theorem is satisfied. Note, however, that the statement of the main theorem is for all abelian varieties in the closure of the locus $\mathcal{P}_{g}$ in $\mathcal{A}_{g}$. To show that condition (A) holds for abelian varieties in the closure, it is enough to note that $(A)$ is an algebraic condition, and thus is valid on the closure of the locus.

When we prove the characterization - the "only if" part of the main theorem - in section 5, there will be no problems with the closure as we will be able to show explicitly that condition $(C)$ (implied by $(A)$ ) exhibits the abelian variety as the Prym for a possibly nodal curve. 


\section{A discrete analog of Novikov-Veselov hierarchy}

In this section we introduce multi-parametric deformations of the Baker-Akhiezer functions and prove that they satisfy a system of difference-differential equations. The compatibility conditions of these equations can be regarded as a discrete analog of the Novikov-Veselov hierarchy $([25])$.

Let $t=\left\{t_{i}^{1}, t_{i}^{2}, i=1,2 \ldots\right\}$ be two sequences of complex numbers (we assume that only finitely many of them are non-zero). We will construct a function $\psi$ on the curve $\Gamma$ with prescribed exponential essential singularities at the points $P_{i}^{ \pm}$controlled by these $t$.

Lemma 3.1 Let $D=D(Z)=\gamma_{1}+\ldots+\gamma_{2 g}$ be an admissible divisor. Then there exists a unique up to a constant factor meromorphic function $\psi_{n, m}(t, P)$ of $P \in \Gamma$, which we call a multi-parametric deformation of the Baker-Akhiezer function, such that

(i) outside of the marked points it has poles only at the points $\gamma_{s}$ of multiplicity not greater than the multiplicity of $\gamma_{s}$ in $D$

(ii) $\psi_{n, m}(t, P)$ has an at most simple pole at $P_{3}^{+}$

(iii) in the local coordinate $k^{-1}$ mapping a small neighborhood of $P_{1}^{ \pm}$to a small disk in $\mathbb{C}$, (with the marked point mapping to zero), it has the power series expansion

$$
\psi_{n, m}(t, P)=k^{\mp n} e^{ \pm \sum_{i} t_{i}^{1} k^{-i}}\left(\sum_{s=0}^{\infty} \xi_{s}^{ \pm}(n, m, t) k^{s}\right),
$$

for some $\xi_{s}^{ \pm}$(notice that this means there is an essential singularity, and the expansion starts from $k^{-n}$ at $P_{1}^{+}$and $k^{n}$ at $P_{1}^{-}$, and goes towards $k^{-\infty}$ )

(iv) in the local coordinate $k^{-1}$ near $P_{2}^{ \pm}$it has the power series expansion

$$
\psi_{n, m}(t, P)=k^{\mp m} e^{ \pm \sum_{i} t_{i}^{2} k^{-i}}\left(\sum_{s=0}^{\infty} \chi_{s}^{ \pm}(n, m, t) k^{s}\right) \text {. }
$$

Proof. This function $\psi_{n, m}$ is given by

$$
\begin{aligned}
\psi_{n, m}(t, P) & =h_{n, m}(t) \frac{\theta\left(A(P)+n U+m V+\nu_{n m} W+Z+\sum_{i}\left(t_{i}^{1} U_{i}^{1}+t_{i}^{2} U_{i}^{2}\right)\right)}{\theta(A(P)+Z)} \times \\
& \times \exp \left(n \Omega_{1}(P)+m \Omega_{2}(P)+\nu_{n m} \Omega_{3}(P)+\sum_{i}\left(t_{i}^{1} \Omega_{i}^{1}(P)+t_{i}^{2} \Omega_{i}^{2}(P)\right)\right),
\end{aligned}
$$

where $\Omega_{1}, \Omega_{2}, \Omega_{3}$ and the vectors $U, V, W$ are as in Lemma $2.2 ; \Omega_{j}^{\mu}$ for $\mu=1,2$ is the abelian integral of the differential $d \Omega_{j}^{\mu}$ which has poles of the form

$$
d \Omega_{j}^{1(2)}= \pm d\left(k^{j}+O(1)\right)
$$


at the punctures $P_{1(2)}^{ \pm}$, holomorphic everywhere else and is uniquely determined by the normalization conditions

$$
\oint_{a_{k}} d \Omega_{j}^{1(2)}=0, \quad k=0, \ldots, 2 g ;
$$

coordinates of the vectors $U_{j}^{1(2)}$ are defined by $b$-periods of these differentials, i.e.

$$
2 \pi i U_{k, j}^{1(2)}=\oint_{b_{k}} d \Omega_{j}^{1(2)}, k=1, \ldots, g
$$

Note that, as before, if $\nu_{n m}=0$ then $\psi_{n, m}$ is in fact holomorphic at $P_{3}^{+}$, and if $\nu_{n m}=1$, then $\psi_{n, m}$ does have a pole at $P_{3}^{+}$, but also has a zero at $P_{3}^{-}$. As before, we normalize $\psi_{n, m}$ by the conditions $(2.25)$.

Notations. In what follows we will deal with formal pseudodifference operators, shifting $n$ and $m$, with coefficients being functions of the variables $n$ and $m$, and of the $t$ 's. From now on when we write functions $f, g, \ldots$ as coefficients of pseudodifference operators, they are meant to be functions of $n, m$ and $t$.

Denote by $\mathcal{R}$ the ring of functions of variables $n, m$, and $t$. We denote by $\mathcal{O}_{1}^{ \pm}$the rings of pseudodifference operators in two variables that are Laurent polynomials in $T_{1}^{ \pm}$, i.e.

$$
\mathcal{O}_{1}^{ \pm}:=\mathcal{R}\left(\left(T_{1}^{\mp}\right)\right)\left[T_{2}, T_{2}^{-1}\right]=\left\{D=\sum_{j=M_{1}}^{M_{2}} \sum_{i=N}^{\infty} r_{i j} T_{1}^{i} T_{2}^{j}\right\}
$$

where $r_{i j} \in \mathcal{R}$. The intersection

$$
\mathcal{O}:=\mathcal{O}_{1}^{+} \cap \mathcal{O}_{1}^{-}=\mathcal{R}\left[T_{1}, T_{1}^{-1}, T_{2}, T_{2}^{-1}\right]
$$

is the ring of difference operators. We further denote $\mathcal{O}_{1,0}^{ \pm}$the ring of pseudodifference operators in one variable that are Laurent polynomials in $T_{1}^{+}$, thought of as subrings of $\mathcal{O}_{1}^{ \pm}$, respectively, i.e.

$$
\mathcal{O}_{1,0}^{ \pm}:=\mathcal{R}\left(\left(T_{1}^{\mp}\right)\right)=\left\{D=\sum_{i=N}^{\infty} r_{i} T_{1}^{i}\right\} .
$$

Finally we denote by $\mathcal{O}_{H}$ the left principal ideal generated by the operator $H=T_{1} T_{2}-$ $u\left(T_{1}-T_{2}\right)-1$, i.e. $\mathcal{O}_{H}:=\mathcal{O} H$, and similarly set $\mathcal{O}_{H}^{ \pm}:=\mathcal{O}_{1}^{ \pm} H$.

Moreover, in doing computations in these rings it is often convenient to compute only a couple highest terms. To this end, we will use for $k>0$ notations $O\left(T_{1}^{-k}\right)=T_{1}^{-k} \mathcal{R}\left[\left[T_{1}^{-1}\right]\right]$ for the operators in $\mathcal{O}_{1}^{+}$only having the terms with $T_{1}^{n}$ for $n \leq-k$, and by $O\left(T_{1}^{k}\right)=T_{1}^{k} \mathcal{R}\left[\left[T_{1}\right]\right]$ - operators in $\mathcal{O}_{1}^{-}$only having terms with $T_{1}^{n}$ for $n \geq k$.

We now want to show that the multi-parametric deformations of the Baker-Akhiezer functions satisfy a hierarchy of difference-differential equations - the result is as follows. 
Proposition 3.2 The Baker-Akhiezer function $\psi=\psi_{n, m}(t, P)$ satisfies (1.1) with $u_{n m}$ as in (1.2), with $Z$ replaced by $Z+\sum_{j}\left(t_{j}^{1} U_{j}^{1}+t_{j}^{2} U_{j}^{2}\right)$ (this can be written as $\left.H \psi=0\right)$. There exist unique difference operators of the form

$$
L_{j}^{(\mu)}=\left(f_{0 j}+\sum_{i=1}^{j-1} f_{i j}^{(\mu)} T_{\mu}^{i}+T_{\mu}^{-i} f_{i j}^{(\mu)}\right)\left(T_{\mu}-T_{\mu}^{-1}\right), \quad \mu=1,2, \quad j=1,2, \ldots
$$

such that the equations

$$
\frac{\partial}{\partial t_{j}^{\mu}} \psi=L_{j}^{(\mu)} \psi
$$

hold.

Proof. The proof of the first statement is identical to that in lemma 2.3. The proof of the statement that there are operators of the form

$$
L_{j}^{(\mu)}=\sum_{i=-j}^{j} g_{i j}^{(\mu)} T_{\mu}^{i}
$$

such that (3.8) hold is standard. Indeed, for each formal series (3.1) there exists a unique operator $L_{j}^{(1)}$ such that

$$
\left(\frac{\partial}{\partial t_{j}^{\mu}}-L_{j}^{(1)}\right) \psi=k^{ \pm n} e^{ \pm \sum_{i} t_{i}^{1} k^{i}}\left(\sum_{s=0}^{\infty} \tilde{\xi}_{s}^{ \pm} k^{-s}\right), \quad \tilde{\xi}_{0}^{+}=0
$$

The coefficients $g_{j}^{(1)}$ of the operator are difference polynomials in terms of the coefficients $\xi_{s}$ of the series (3.1). Now note that the left-hand-side of (3.10) satisfies all the properties that $\psi$ satisfies, and thus must be proportional to it. However, since $\tilde{\xi}_{0}^{+}=0$, the constant of proportionality must be equal to zero, and thus the left-hand-side vanishes as desired. The same arguments proves the existence of $L_{j}^{(2)}$.

It remains to show that the operators $L_{j}^{(\mu)}$ have the form (3.7). This is a matter of showing that the coefficients of $L_{j}^{(\mu)}$ satisfy certain identities, i.e. that the generally constructed $g_{i j}^{(\mu)}$ for $-j \leq i \leq j$ can in fact be expressed in terms of $f_{i j}^{(\mu)}$ for $1 \leq i \leq j-1$. One easily checks that if $L_{j}^{(\mu)}$ is given by (3.7), then its operator formal adjoint satisfies

$$
\left(L_{j}^{(\mu)}\right)^{*}=-\left(T_{\mu}-T_{\mu}^{-1}\right) L_{j}^{(\mu)}\left(T_{\mu}-T_{\mu}^{-1}\right)^{-1} .
$$

This equation is in fact equivalent to (3.7), as it determines the coefficients of all the negative powers of $T_{\mu}$ uniquely, given the coefficients of the positive powers. It thus remains to prove this identity.

We denote by $\psi^{\sigma}$ the composition $\psi^{\sigma}(P)=\psi(\sigma(P))$ - notice that by (3.1) we know the expansion of both $\psi$ and $\psi^{\sigma}$ near $P_{1}^{ \pm}$. Now consider the differential $\psi^{\sigma}\left(T_{\mu}^{n}\left(T_{\mu}-T_{\mu}^{-1}\right) \psi\right) d \Omega$, 
where, as before, $d \Omega$ is a holomorphic differential having zeros at poles of $\psi$ and $\psi^{\sigma}$. Then this expression is a meromorphic differential on $\Gamma$ which a priori has poles only at $P_{\mu}^{ \pm}$and $P_{3}^{ \pm}$. Due to normalization $(2.25)$ it is holomorphic at the punctures $P_{3}^{ \pm}$- the pole of $\psi$ cancels with the zero of $\psi^{\sigma}$ and vice versa. Therefore, for $n>0$ this differential has a pole only at $P_{\mu}^{+}$, and hence its residue at this point must vanish:

$$
\operatorname{res}_{P_{\mu}^{+}}\left(\psi^{\sigma}\left(T_{\mu}^{n}\left(T_{\mu}-T_{\mu}^{-1}\right) \psi\right) d \Omega\right)=0, \quad \forall n>0 .
$$

The normalization (2.25) implies also

$$
\operatorname{res}_{P_{\mu}^{+}}\left(\psi^{\sigma}\left(T_{\mu} \psi\right) d \Omega\right)=1 .
$$

Equations $(3.12,3.13)$ recurrently define coefficients of the power series expansion of $\psi^{\sigma} d \Omega$ at $P_{\mu}^{+}$in terms of the coefficients of the power series for $\psi$. The corresponding expressions can be explicitly written in terms of the so-called wave operator.

We first observe that in the ring $\mathcal{O}_{1,0}^{+}$there exists a unique pseudo-difference operator

$$
\Phi=\sum_{s=0}^{\infty} \varphi_{s} T_{1}^{-s}
$$

such that the expansion $(3.1)$ of $\psi$ at $P_{1}^{+}$is equal to

$$
\psi=\Phi k^{n} e^{\sum_{i} t_{i}^{1} k^{i}}
$$

Indeed, this identity gives a unique way to determine the coefficients $\varphi_{s}$ recursively.

Lemma 3.3 The following identity holds:

$$
\psi^{\sigma} d \Omega=\left(k^{-n} e^{-\sum_{i} t_{i}^{1} k^{i}}\left(T_{1}-T_{1}^{-1}\right) \Phi^{-1}\left(T_{1}-T_{1}^{-1}\right)^{-1}\right) \frac{d k}{k^{2}-1}
$$

Here and below the right action of pseudo-difference operators is defined as the formal adjoint action, i.e we set $f T=T^{-1} f$.

Proof. Recall that by definition the residue of a pseudo-differential operator $D=\sum_{s} d_{s} T^{s}$ is $\operatorname{res}_{T} D:=d_{0}$. It is easy to check - by verifying that this holds for the basis, i.e. checking this for $D_{1}=T_{1}^{a}$ and $D_{2}=T_{1}^{b}$ — that for any two pseudo-differential operators $D_{1}, D_{2}$ we have

$$
\operatorname{res}_{k}\left(k^{-n} e^{-\sum_{i} t_{i}^{1} k^{i}} D_{1}\right)\left(D_{2} k^{n} e^{\sum_{i} t_{i}^{1} k^{i}}\right) d \ln k=\operatorname{res}_{T}\left(D_{2} D_{1}\right)
$$

The last equation implies that

$$
\begin{gathered}
\operatorname{res}_{k}\left(k^{-n} e^{-\sum_{i} t_{i}^{1} k^{i}}\left(T_{1}-T_{1}^{-1}\right) \Phi^{-1}\left(T_{1}-T_{1}^{-1}\right)^{-1}\right)\left(T_{1}^{n}\left(T_{1}-T_{1}^{-1}\right) \psi\right) \frac{d k}{k^{2}-1}= \\
\operatorname{res}_{k}\left(k^{-n} e^{-\sum_{i} t_{i}^{1} k^{i}} \Phi^{-1}\left(T_{1}-T_{1}^{-1}\right)^{-1}\right)\left(T_{1}^{n}\left(T_{1}-T_{1}^{-1}\right) \Phi k^{n} e^{\sum_{i} t_{i}^{1} k^{i}}\right) d \ln k=\operatorname{res}_{T} T_{1}^{n}=\delta_{n, 0},
\end{gathered}
$$


i.e. the formal series defined by the right hand side of (3.16) satisfies the equations $(3.12,3.13)$, which are the defining equations (by solving term by term, see above) for $\psi^{\sigma} d \Omega$.

Now we are ready to complete the proof that the adjoints of $L_{j}^{\mu}$ satisfy (3.11), thus proving proposition 3.2. Consider the pseudo-difference operator

$$
\mathcal{L}:=\Phi T_{1} \Phi^{-1}
$$

for which $\psi$ is an eigenvector: indeed

$$
\mathcal{L} \psi=\Phi T_{1} k^{n} e^{\sum_{i} t_{i}^{1} k^{i}}=\Phi k^{n+1} e^{\sum_{i} t_{i}^{1} k^{i}}=k \psi .
$$

Considering the expansion of (3.8) in a neighborhood of $P_{1}^{+}$, we see that the positive parts of the pseudo-difference operators $L_{j}^{(1)}$ and $\mathcal{L}^{j}$ coincide:

$$
\left(L_{j}^{(1)}\right)_{+}=\mathcal{L}_{+}^{j}
$$

(where by the positive part of a pseudo-difference operator $D=\sum_{s} d_{s} T^{s}$ we mean $D_{+}:=$ $\left.\sum_{s>0} d_{s} T^{s}\right)$.

The differential $d \Omega$ is independent of $n$. Therefore, from (3.16) it follows that the operator

$$
\widetilde{\mathcal{L}}:=\left(T_{1}-T_{1}^{-1}\right)^{-1} \mathcal{L}^{*}\left(T_{1}-T_{1}^{-1}\right) .
$$

has $\psi^{\sigma}$ as an eigenfunction:

$$
\widetilde{\mathcal{L}} \psi^{\sigma}=k \psi^{\sigma} .
$$

Equation (3.8) considered in the neighborhood of $P_{1}^{-}$implies that the negative parts of $L_{j}^{(1)}$ and $\widetilde{\mathcal{L}}^{j}$ coincide,

$$
\left(L_{j}^{(1)}\right)_{-}=-\widetilde{\mathcal{L}}_{-}^{j}
$$

The last two equations prove (3.11) and then (3.7) for $\mu=1$. The case $\mu=2$ is analogous, and the proposition is thus proven.

Corollary 3.4 The operators $H$ and $L_{j}^{(\mu)}$ satisfy the equations

$$
\frac{\partial}{\partial t_{j}^{\mu}} H \equiv\left[L_{j}^{\mu}, H\right] \bmod \mathcal{O}_{H},
$$

Proof. It is easy to show that the ideal of pseudodifference operators $D$ such that $D \psi=0$ is $\mathcal{O}_{H}$. From (3.8) it follows that

$$
\left(\partial_{t_{j}^{\mu}} H-\left[L_{j}^{\mu}, H\right]\right) \psi=0 .
$$

Hence, the right and left hand sides of (3.23) are equal in the factor-ring $\mathcal{O} / \mathcal{O}_{H}$ 
It will be shown below that the system of non-linear equations (3.23) can be regarded as a discrete analog of Novikov-Veselov hierarchy. The basic equation of this hierarchy - the discrete analog of the Novikov-Veselov equation — is given by (3.23) for $j=1$. The operator $L_{1}^{(1)}$ is of the form

$$
L_{1}^{(1)}=v\left(T_{1}-T_{1}^{-1}\right) .
$$

Equation (3.23) is equivalent to the system of two equations for the two functions $u=$ $u_{n, m}(t), v=v_{n, m}(t)$ :

$$
\begin{gathered}
v\left(\mathbf{t}_{1}^{-1} u\right)=u\left(\mathbf{t}_{2} v\right) \\
\partial_{t} u=\left[\left(\mathbf{t}_{1} \mathbf{t}_{2} v\right)\left(\mathbf{t}_{1} u\right)-u\left(\mathbf{t}_{2} v\right)\right] u-\left[\mathbf{t}_{1} \mathbf{t}_{2} v-v\right]
\end{gathered}
$$

\section{The discrete Novikov-Veselov hierarchy.}

The discrete analog of the Novikov-Veselov hierarchy is of an independent interest. In what follows we consider only the part of the hierarchy corresponding to "times" $t_{j}:=t_{j}^{1}$, and set all $t_{j}^{2}=0$.

Let us write out this part of the hierarchy in a closed form. We think of it as a system of evolution equations on the following space

$$
\mathcal{S}:=\left\{H, \mathcal{L} \mid H=T_{1} T_{2}-u\left(T_{1}-T_{2}\right)-1, \mathcal{L}=\sum_{i=0}^{\infty} v_{i} T_{1}^{-i+1}\right\}
$$

satisfying

$$
[H, \mathcal{L}] \equiv 0 \bmod \mathcal{O}_{H}^{+},
$$

and such that moreover $u$ and $v_{0}$ are of the form

$$
u=C \frac{\left(\mathbf{t}_{1} \tau\right)\left(\mathbf{t}_{2} \tau\right)}{\left(\mathbf{t}_{1} \mathbf{t}_{2} \tau\right) \tau}, \quad v_{0}=\frac{\left(\mathbf{t}_{1} \tau\right)\left(\mathbf{t}_{1}^{-1} \tau\right)}{\tau^{2}}
$$

where $C$ is a constant and $\tau=\tau(n, m)$ is some function.

The meaning of $(3.28)$ is as follows. A priori the operator $[H, \mathcal{L}]$ has a unique representation of the form

$$
[H, \mathcal{L}]=\left(\sum_{s=0}^{\infty} h_{s} T_{1}^{-s+2}\right)+D H,
$$

with $D \in \mathcal{O}_{1}^{+}$. Therefore, the constraint (3.28) is equivalent to equations $h_{s}=0$. The first of these equations $h_{0}=0$ is an equation for $u$ and $v_{0}$, which is automatically satisfied due to $(3.29)$.

By a direct computation of the series expansion of $[H, \mathcal{L}]$ it is easy to see that equations $h_{s}=0$ for $s>0$ have the form

$$
\left(\mathbf{t}_{2} v_{s}\right)\left(\mathbf{t}_{1}^{-s} u\right)-\left(\mathbf{t}_{1}^{-1} u\right) v_{s}=R_{s}\left(\tau, v_{1}, \ldots, v_{s-1}\right),
$$

where $R_{s}$ is some difference polynomial. They recurrently define $v_{s}(n, m)$, if "the initial data" $\left.v_{s}\right|_{m=0}$ are fixed. Therefore, the space $\mathcal{S}$ of operators $H, \mathcal{L}$ with the leading coefficients 
$u, v_{0}$ of the form (3.29) satisfying (3.28) can be identified with the space of one function of two variables and infinite number of functions of one variable, i.e. $\left\{\tau(n, m), v_{s}(n), s>0\right\}$.

Our next goal is to define on $\mathcal{S}$ a hierarchy of commuting flows. Any operator in $\mathcal{O}_{1,0}^{+}$, and in particular $\mathcal{L}^{j}$, has a unique representation in the form

$$
\mathcal{L}^{j}=\sum_{i=-\infty}^{j-1} f_{i j} T_{1}^{i}\left(T_{1}-T_{1}^{-1}\right)
$$

Then the formula (3.7) with $\mu=1$ defines a unique operator $L_{j}:=L_{j}^{(1)}$ such that (3.19) holds, and also satisfying the condition (3.11) with $\mu=1$ for the adjoint.

Theorem 3.5 The equations

$$
\partial_{t_{j}} \mathcal{L}=\left[L_{j}, \mathcal{L}\right], \quad \partial_{t_{j}} H \equiv\left[L_{j}, H\right] \bmod \mathcal{O}_{H}
$$

define commuting flows on the space $\mathcal{S}$.

Proof. Note that the highest power of $T_{1}$ in $\mathcal{L}$ is $T_{1}$, and $\partial_{t_{j}} H=\left(\partial_{t_{j}} u\right)\left(T_{1}-T_{2}\right)$. Thus in order to show that equations (3.32) are well-defined we need to prove the following

(a) $\left[L_{j}, \mathcal{L}\right]$ is of degree not greater than 1 ;

(b) $\left[L_{j}, H\right] \equiv a_{j}\left(T_{1}-T_{2}\right) \bmod \mathcal{O}_{H}$;

(c) the corresponding equations for $v_{0}$ and $u$ are consistent with the ansatz (3.29).

The proof of (a) is standard. We compute

$$
L_{j}=\mathcal{L}^{j}+F_{j}+F_{j}^{1} T_{1}^{-1}+O\left(T^{-2}\right),
$$

where

$$
F_{j}=\mathbf{t}_{1}^{-1} f_{1, j}-f_{-1, j}, F_{j}^{1}=\mathbf{t}_{1}^{-2} f_{2, j}-f_{-2, j} .
$$

Using $\left[\mathcal{L}, \mathcal{L}^{j}\right]=0$, we get

$$
\left[L_{j}, \mathcal{L}\right]=\left[F_{j}+O\left(T_{1}^{-1}\right), \mathcal{L}\right]=\left(F_{j}-\mathbf{t}_{1} F_{j}\right) v_{0} T_{1}+O(1)
$$

thus proving (a). Note also that by comparing the leading coefficients we obtain

$$
\frac{\partial}{\partial t_{j}} \ln v_{0}=F_{j}-\mathbf{t}_{1} F_{j}
$$

The proof of (b) is much harder. The difference operator $H L_{j}$ is of order 1 in $T_{2}$. Hence it has a unique representation of the form

$$
H L_{j}=D_{1}-a_{j} T_{2}+D H,
$$

where $D \in \mathcal{O}$ and $D_{1} \in \mathcal{O}_{1,0}$. 
Our next goal is to show that $D_{1}$ is of degree 1 in $T_{1}$, i.e. has the form $D_{1}=b_{j} T_{1}+c_{j}$. From the equation $T_{1}^{-1} H \equiv 0 \bmod \mathcal{O}_{H}$ we get

$$
T_{2}=\mathbf{t}_{1}^{-1} u+T_{1}^{-1}-\mathbf{t}_{1}^{-1} u T_{1}^{-1} T_{2}=\mathbf{t}_{1}^{-1} u+\left(1-\mathbf{t}_{1}^{-1} u \mathbf{t}^{-2} u\right) T^{-1}+O\left(T_{1}^{-2}\right) .
$$

Equations $\left[\mathcal{L}^{j}, H\right]=0$ and (3.33) imply that in $\mathcal{O}_{H}^{+}$the left hand side of (3.36) is equal to

$$
\begin{gathered}
H L_{j}=\left(\left(\mathbf{t}_{1} \mathbf{t}_{2} F_{j}-\mathbf{t}_{1} F_{j}\right) u\right) T_{1}+ \\
+\left(\left(1-u \mathbf{t}_{1}^{-1} u\right) \mathbf{t}_{1} \mathbf{t}_{2} F_{j}+\left(u \mathbf{t}_{1}^{-1} u\right) \mathbf{t}_{2} F_{j}-F_{j}+\left(\mathbf{t}_{1}^{-1} u\right) \mathbf{t}_{1} \mathbf{t}_{2} F_{j}^{1}-u \mathbf{t}_{1} F_{j}^{1}\right)+O\left(T^{-1}\right)
\end{gathered}
$$

Substituting this expression and the formula for $T_{2}$ in (3.36), we get $D_{1}=b_{j} T_{1}+c+O\left(T_{1}^{-1}\right)$, where

$$
\begin{gathered}
b_{j}:=\left(\mathbf{t}_{1} \mathbf{t}_{2} F_{j}-\mathbf{t}_{1} F_{j}\right) u \\
c_{j}:=a_{j} \mathbf{t}_{1}^{-1} u+\left(1-u \mathbf{t}_{1}^{-1} u\right) \mathbf{t}_{1} \mathbf{t}_{2} F_{j}+\left(u \mathbf{t}_{1}^{-1} u\right) \mathbf{t}_{2} F_{j}-F_{j}+\left(\mathbf{t}_{1}^{-1} u\right) \mathbf{t}_{1} \mathbf{t}_{2} F_{j}^{1}-u \mathbf{t}_{1} F_{j}^{1}
\end{gathered}
$$

Now we are going to compute the left and the right hand sides of (3.36) in $\mathcal{O}_{H}^{-}$. Indeed, in $\mathcal{O}_{1}^{-}$we have

$$
L_{j}=-\widetilde{\mathcal{L}}^{j}-\widetilde{F}_{j}-\widetilde{F}_{j}^{1} T_{1}+O\left(T_{1}^{2}\right),
$$

where, as before, $\tilde{\mathcal{L}}=\left(T_{1}-T_{1}^{-1}\right)^{-1} \mathcal{L}^{*}\left(T_{1}-T_{1}^{-1}\right)$. If $f_{i j}$ are coefficients of $\widetilde{L}$ in $(3.31)$, then

$$
\widetilde{\mathcal{L}}^{j}=-\sum_{i=-\infty}^{j-1} T_{1}^{-i} \cdot f_{i j} \cdot\left(T_{1}-T_{1}^{-1}\right) .
$$

Hence,

$$
\widetilde{F}_{j}=\mathbf{t}_{1} F_{j}=f_{1, j}-\mathbf{t}_{1} f_{-1, j}, \widetilde{F}_{j}^{1}=\mathbf{t}_{1}^{2} F_{j}^{1}=f_{2, j}-\mathbf{t}_{2} f_{-2, j} .
$$

In order to proceed we now need the following statement.

Lemma 3.6 If (3.28) is satisfied, then the equation

$$
[H, \widetilde{\mathcal{L}}] \equiv 0 \bmod \mathcal{O}_{H}^{-}
$$

holds.

Proof. We will prove the lemma by inverting the arguments used above in the proof of Lemma 3.2. First, for a pair of operators $\mathcal{L}$ and $H$ satisfying (3.28) we introduce a formal solution $\psi=\psi_{n m}$ of equations

$$
\mathcal{L} \psi=k \psi, \quad H \psi=0
$$

of the form

$$
\psi_{n m}=k^{n}\left(\sum_{s=0}^{\infty} \xi_{s}(n, m) k^{-s}\right) .
$$

Substitution of (3.46) into (3.45) gives a system of difference equations, which recurrently define $\xi_{s}$. They have the form

$$
\left(T_{2} \xi_{s+1}\right)-u \xi_{s+1}=\xi_{s}-u\left(T_{2} \xi_{s}\right), \quad v_{0}\left(T_{1} \xi_{s+1}\right)-\xi_{s+1}=\tilde{R}_{s}
$$


where $\tilde{R}_{s}$ are explicit expression linear in the coefficients $v_{r}$ of $\mathcal{L}$ and difference polynomial in $\xi_{r}, r<s$. If $u, v_{0}$ are of the form (3.29), then the first equation for $s=-1$ is satisfied by

$$
\xi_{0}=\frac{\mathbf{t}_{1}^{-1} \tau}{\tau}
$$

The compatibility condition of equations (3.47) is equivalent to (3.28). These equations uniquely define $\xi_{s+1}$ for all $(n, m)$, if the initial data $\xi_{s+1}(0,0)$ for $(3.47)$ is fixed. Therefore, the solution $\psi$ is unique up to multiplication by a $(n, m)$-independent Laurent series in the variable $k$.

The function $\psi$ defines a unique operator $\Phi$ of the form (3.14) such that equation (3.15) holds (with $t_{i}=0$ ). Now we define a formal series

$$
\psi^{\sigma}=k^{-n}\left(\sum_{s=0}^{\infty} \xi_{s}^{\sigma}(n, m) k^{-s}\right), \xi_{0}^{\sigma}=\frac{\mathbf{t}_{1} \tau}{\tau}
$$

by the formula

$$
\psi^{\sigma}=\left(\left(T_{1}-T_{1}^{-1}\right) \Phi^{-1}\left(T_{1}-T_{1}^{-1}\right)^{-1}\right)^{*} k^{-n} .
$$

This formal series is an eigenfunction of the operator $\widetilde{L}$, i.e. $\widetilde{\mathcal{L}} \psi^{\sigma}=k \psi^{\sigma}$. Therefore, in order to prove (3.44) it is sufficient to prove that $H \psi^{\sigma}=0$.

From equations $(3.29,3.48)$ it follows that

$$
\widetilde{\psi}^{\sigma}:=H \psi^{\sigma}=k^{-n}\left(\sum_{s=1}^{\infty} \widetilde{\xi}^{\sigma}(n, m) k^{-s}\right)
$$

Hence, to prove that $\tilde{\psi}^{\sigma}=0$ it is enough to show that

$$
\left[\widetilde{\psi}^{\sigma}\left(T_{1}^{j} \psi\right)\right]_{R}:=\operatorname{res}_{k}\left(\frac{\tilde{\psi}^{\sigma}\left(T_{1}^{j} \psi\right) d k}{k^{2}-1}\right)=0, \quad \forall j \geq 2
$$

From the definition of $\psi^{\sigma}$ it follows that

$$
\left[\psi^{\sigma}\left(\mathbf{t}_{1}^{2 j} \psi\right)\right]_{R}=0, \quad\left[\psi^{\sigma}\left(\mathbf{t}_{1}^{2 j+1} \psi\right)\right]_{R}=1, \quad j \geq 0
$$

(compare to $(3.12,3.13))$. Using the equation $H \psi=0$, we get

$$
\left.\mathbf{t}_{2}\left[\psi^{\sigma} t^{2 j} \psi\right]_{R}=\left(\mathbf{t}_{1}^{2 j-1} u\right)\left[\mathbf{t}_{2} \psi^{\sigma} t^{2 j-1} \psi\right)\right]_{R}-\left(\mathbf{t}_{1}^{2 j-1} u\right) \mathbf{t}_{2}\left[\psi^{\sigma} t^{2 j-1} \psi\right]_{R}+\left[\mathbf{t}_{2} \psi^{\sigma} t^{2 j-1}\right]_{R}
$$

Then, by induction, it is easy to show that (3.53) and (3.54) imply

$$
\left.\left[\mathbf{t}_{2} \psi^{\sigma} t^{2 j+2} \psi\right)\right]_{R}=1-\prod_{i=0}^{2 j+1}\left(\mathbf{t}_{1}^{i} u\right)^{-1}, \quad\left[\mathbf{t}_{2} \psi^{\sigma} t^{2 j+1} \psi\right]_{R}=\prod_{i=0}^{2 j}\left(\mathbf{t}_{1}^{i} u\right)^{-1}, \quad j \geq 0 .
$$

Direct substitution of (3.55) into (3.52) completes the proof of the lemma. Now we compute both sides of (3.36): 


$$
T_{2} \equiv \frac{1}{u}+\left(1-\frac{1}{u \mathbf{t}_{1} u}\right) T_{1}^{1}+O\left(T_{1}^{2}\right) \bmod \mathcal{O}_{H}^{-}
$$

Equations (3.41) and (3.44) imply $\left[L_{j}, H\right]=H\left(\widetilde{F}_{j}+\widetilde{F}_{j}^{1} T_{1}+O\left(T_{1}^{2}\right)\right) \in \mathcal{O}_{H}^{-}$. Therefore, the operator $D_{1}$ in (3.36) has no negative powers of $T_{1}$, and hence, it is indeed of the form $b_{j} T_{1}+c_{j}$.

Straightforward computations of the first two coefficients of $\left[L_{j}, H\right]$ give the following formulae

$$
\begin{gathered}
c_{j}-\frac{a_{j}}{u}=\widetilde{F}_{j}-\mathbf{t}_{2} \widetilde{F}_{j} \\
\left(1-\frac{1}{u \mathbf{t}_{1} u}\right) a_{j}-b_{j}=\frac{1}{\mathbf{t}_{1} u}\left(\mathbf{t}_{1} \mathbf{t}_{2} \widetilde{F}_{j}+\left(u \mathbf{t}_{1} u-1\right) \mathbf{t}_{2} \widetilde{F}_{j}-\left(u \mathbf{t}_{1} u\right) \mathbf{t}_{1} \widetilde{F}_{j}-\mathbf{t}_{1} u \widetilde{F}_{j}^{1}+u \mathbf{t}_{2} \widetilde{F}_{j}^{1}\right)
\end{gathered}
$$

From $(3.39,3.43)$ and $(3.57)$ we get the equations

$$
c_{j} u=\left(a_{j}-b_{j}\right)
$$

and then

$$
c_{j}\left(u \mathbf{t}_{1} u-1\right)=\mathbf{t}_{1} \mathbf{t}_{2} \widetilde{F}_{j}+u \mathbf{t}_{1} u\left(\mathbf{t}_{2} \widetilde{F}_{j}-\mathbf{t}_{1} \widetilde{F}_{j}\right)-\mathbf{t}_{1} u \widetilde{F}_{j}^{1}+u \mathbf{t}_{2} \widetilde{F}_{j}^{1}-\widetilde{F}_{j}
$$

In order to complete the proof of (b) it is enough now to show that the right hand side of (3.60) is zero. For that we need the following

Lemma 3.7 The equations

$$
\begin{gathered}
\widetilde{\mathcal{F}}:=-k+\left(k^{2}-1\right) \sum_{j=1}^{\infty} \widetilde{F}_{j} k^{-j-1}=\left(\mathbf{t}_{1} \psi^{\sigma}\right) \psi-\psi^{\sigma}\left(\mathbf{t}_{1} \psi\right), \\
\widetilde{\mathcal{F}}^{1}:=-\frac{\left(\mathbf{t}_{1} \tau\right)^{2}}{\tau \mathbf{t}_{2} \tau} k^{2}+\left(k^{2}-1\right) \sum_{j=1}^{\infty} \widetilde{F}_{j}^{1} k^{-j-1}=\psi^{\sigma}\left(\mathbf{t}_{1}^{2} \psi\right)-\left(\mathbf{t}_{1}^{2} \psi^{\sigma}\right) \psi
\end{gathered}
$$

hold.

Proof. The expression for the leading coefficients of $\widetilde{\mathcal{F}}_{j}$ and $\widetilde{\mathcal{F}}_{j}^{1}$ follows from (3.48) and (3.50). In order to prove (3.61) we need to show that

$$
\widetilde{F}_{j}=\operatorname{res}_{k}\left(\left[\left(\mathbf{t}_{1} \psi^{\sigma}\right) \psi-\psi^{\sigma}\left(\mathbf{t}_{1} \psi\right)\right] \frac{k^{j} d k}{k^{2}-1}\right)=\operatorname{res}_{k}\left(\left[\left(T_{1} \psi^{\sigma}\right)\left(\mathcal{L}^{j} \psi\right)-\psi^{\sigma}\left(T_{1} \mathcal{L}^{j} \psi\right)\right] \frac{d k}{k^{2}-1}\right)
$$

From (3.50), using the relation (3.17), we see that the right hand of (3.64) is equal to

$$
\operatorname{res}_{T}\left(\left(\mathcal{L}^{j} T_{1}^{-1}-T_{1} \mathcal{L}^{j}\right)\left(T_{1}-T_{1}^{-1}\right)^{-1}\right)=f_{1, j}-\mathbf{t}_{1} f_{-1, j}
$$

which proves (3.61). The proof of (3.62) is identical. 
From (3.61) and the equation $H \psi=0$ it follows that

$$
\mathbf{t}_{2} \widetilde{\mathcal{F}}=-\mathcal{A}\left\{\left(\mathbf{t}_{2} \psi^{\sigma}\left(u \mathbf{t}_{1} \psi-u \mathbf{t}_{2} \psi+\psi\right)\right\}=-\mathcal{A}\left\{\left(\mathbf{t}_{2} \psi^{\sigma}\left(u \mathbf{t}_{1} \psi+\psi\right)\right\} .\right.\right.
$$

Here and below $\mathcal{A}\{\cdot\}$ stands for the antisymmetrization of the corresponding expression with respect to the interchange of $\psi^{\sigma}$ and $\psi$.

In the same way we get

$$
\begin{gathered}
\mathbf{t}_{1} \mathbf{t}_{2} \widetilde{\mathcal{F}}=-\mathcal{A}\left\{\left(u \mathbf{t}_{1} \psi^{\sigma}-u \mathbf{t}_{2} \psi^{s}+\psi^{\sigma}\right) \mathbf{t}_{1}^{2} \mathbf{t}_{2} \psi\right\}= \\
-\left(u \mathbf{t}_{1} u\right) \mathbf{t}_{1} \mathcal{A}\left\{\psi^{\sigma}\left(\mathbf{t}_{1} \psi-\mathbf{t}_{2} \psi\right)\right\}-u \mathbf{t}_{2} \widetilde{\mathcal{F}}^{1}-\mathcal{A}\left\{\psi^{\sigma}\left(\mathbf{t}_{1}\left(u \mathbf{t}_{1} \psi-u \mathbf{t}_{2} \psi+\psi\right)\right\}\right.
\end{gathered}
$$

Further direct use of the equation $H \psi=0$ and (3.65) finally gives the equation

$$
\mathbf{t}_{1} \mathbf{t}_{2} \widetilde{\mathcal{F}}+u \mathbf{t}_{1} u\left(\mathbf{t}_{1} \widetilde{\mathcal{F}}-\mathbf{t}_{2} \widetilde{\mathcal{F}}\right)-\mathbf{t}_{1} u \widetilde{\mathcal{F}}^{1}+u \mathbf{t}_{2} \widetilde{\mathcal{F}}^{1}-\widetilde{\mathcal{F}}=0
$$

The proof of $(b)$ is complete. The comparison of the coefficients at $T_{1}$ in the left and the right hand sides of (3.32) gives

$$
\partial_{t_{j}} \ln u=b_{j}=\mathbf{t}_{2} \widetilde{F}_{j}-\widetilde{F}_{j}
$$

Now we are going to prove $(c)$ and derive the evolution equation for $\tau$. The left and right action of pseudo-difference operators are formally adjoint, i.e., for any two operators the equality $\left(k^{-x} \mathcal{D}_{1}\right)\left(\mathcal{D}_{2} k^{x}\right)=k^{-n}\left(\mathcal{D}_{1} \mathcal{D}_{2} k^{n}\right)+\left(T_{1}-1\right)\left(k^{-x}\left(\mathcal{D}_{3} k^{x}\right)\right)$ holds. Here $\mathcal{D}_{3}$ is a pseudo-difference operator whose coefficients are difference polynomials in the coefficients of $\mathcal{D}_{1}$ and $\mathcal{D}_{2}$. Therefore, from (3.61) and (3.50) it follows that

$$
\widetilde{\mathcal{F}}^{0}=-k-\left(T_{1}-1\right)\left(\left(k^{2}-1\right) \sum_{s=2}^{\infty} Q_{j} k^{-j}\right)
$$

where the coefficients of the series $Q$ are difference polynomials in the coefficients of the wave operator $\Phi$. Equation (3.68) implies that

$$
\widetilde{F}_{j}=\left(1-T_{1}\right) Q_{j}=Q_{j}-\mathbf{t}_{1} Q_{j} .
$$

Taking into account the ansatz (3.29), we see that equations (3.35) and (3.69) are equivalent to one equation for the function $\tau$

$$
\partial_{t_{j}} \ln \tau=Q_{j}
$$

Remark 3.8 It is necessary to mention that the $Q_{j}$ are defined only up to an additive term that is invariant under $T_{1}$. This ambiguity reflects the fact that the ansatz (3.29) is invariant under the transformation

$$
\tau(n, m) \longmapsto f(m) \tau(n, m)
$$

where $f(m)$ is an arbitrary function.

Equation (3.70) completes the proof of the statement that equations (3.32) are well-defined. The proof of the statement that the corresponding flows on $\mathcal{S}$ commute with each other is standard. 


\section{Bloch (quasi-periodic) wave solutions.}

To begin with let us prove the implication $(A) \Rightarrow(C)$ in the main theorem. As it was mentioned above this does not require the knowledge of the explicit theta-functional form of the function $\psi$. For the first time an implication of this kind was proved in [1].

Throughout this section $\nu=0,1$ and is considered as an element of the group $\mathbb{Z}_{2}=\mathbb{Z} / 2 \mathbb{Z}$.

Lemma 4.1 Let $V \in \mathbb{C}^{d}$, and let $\tau_{n}^{\nu}(z)$ for $n \in \mathbb{N}, \nu \in \mathbb{Z}_{2}$ be two sequences of holomorphic functions on $\mathbb{C}^{d}$ such that each divisor $\mathcal{T}_{n}^{\nu}:=\left\{z \in \mathbb{C}^{d}: \tau_{n}^{\nu}(z)=0\right\}$ is not invariant as a set under the shift by $V$, i.e. $\mathcal{T}_{n}^{\nu} \neq \mathcal{T}_{n}^{\nu}+V$. Suppose that the system of equations (considered as a joint system for $\nu=0$ and $\nu=1$, intertwining $\psi^{0}$ and $\psi^{1}$ )

$$
\psi_{n+1}^{\nu}(z+V)-u_{n}^{\nu}(z)\left(\psi_{n+1}^{\nu+1}(z)-\psi_{n}^{\nu+1}(z+V)\right)-\psi_{n}^{\nu}(z)=0
$$

where

$$
u_{n}^{\nu}(z)=C \frac{\tau_{n+1}^{\nu+1}(z) \tau_{n}^{\nu+1}(z+V)}{\tau_{n+1}^{\nu}(z+V) \tau_{n}^{\nu}(z)}
$$

has solutions $\psi_{n}^{\nu}$ of the form

$$
\psi_{n}^{\nu}(z)=\frac{\alpha_{n}^{\nu}(z)}{\tau_{n}^{\nu}(z)}
$$

where $\alpha_{n}^{\nu}$ is a holomorphic function. Then the equation

$$
\begin{gathered}
\tau_{n+1}^{\nu+1}\left(z_{n}^{\nu}\right) \tau_{n}^{\nu+1}\left(z_{n}^{\nu}+V\right) \tau_{n-1}^{\nu}\left(z_{n}^{\nu}-V\right)+\tau_{n+1}^{\nu}\left(z_{n}^{\nu}+V\right) \tau_{n}^{\nu+1}\left(z_{n}^{\nu}-V\right) \tau_{n-1}^{\nu+1}\left(z_{n}^{\nu}\right) \\
=\left(\tau_{n+1}^{\nu+1}\left(z_{n}^{\nu}\right) \tau_{n}^{\nu+1}\left(z_{n}^{\nu}-V\right) \tau_{n-1}^{\nu}\left(z_{n}^{\nu}+V\right)+\tau_{n+1}^{\nu}\left(z_{n}^{\nu}-V\right) \tau_{n}^{\nu+1}\left(z_{n}^{\nu}+V\right) \tau_{n-1}^{\nu+1}\left(z_{n}^{\nu}\right)\right) C^{2}
\end{gathered}
$$

is valid $\forall n, \nu, \forall z_{n}^{\nu} \in \mathcal{T}_{n}^{\nu}$.

Proof. Let $I_{n}^{\nu}(z)$ be the left hand side of (4.1). A priori it may have poles at the divisors $\mathcal{T}_{n}^{\nu}$ and $\mathcal{T}_{n+1}^{\nu}-V$. The vanishing of the residue of $I_{n}^{\nu}$ at $\mathcal{T}_{n}^{\nu}$ implies

$$
\psi_{n+1}^{\nu+1}\left(z_{n}^{\nu}\right)-\psi_{n}^{\nu+1}\left(z_{n}^{\nu}+V\right)=-\alpha_{n}^{\nu}\left(z_{n}^{\nu}\right) \frac{\tau_{n+1}^{\nu}\left(z_{n}^{\nu}+V\right)}{\tau_{n+1}^{\nu+1}\left(z_{n}^{\nu}\right) \tau_{n}^{\nu+1}\left(z_{n}^{\nu}+V\right)} C^{-1}
$$

while the vanishing of the residue of $I_{n-1}^{\nu}$ at $\mathcal{T}_{n-1}^{\nu}-V$ implies

$$
\psi_{n}^{\nu+1}\left(z_{n}^{\nu}-V\right)-\psi_{n-1}^{\nu+1}\left(z_{n}^{\nu}\right)=\alpha_{n}^{\nu}\left(z_{n}^{\nu}\right) \frac{\tau_{n-1}^{\nu}\left(z_{n}^{\nu}-V\right)}{\tau_{n}^{\nu+1}\left(z_{n}^{\nu}-V\right) \tau_{n-1}^{\nu+1}\left(z_{n}^{\nu}\right)} C^{-1} .
$$

On the other hand, the evaluation of $I_{n}^{\nu+1}$ at the divisor $\mathcal{T}_{n}^{\nu}-V$ implies

$$
\psi_{n+1}^{\nu+1}\left(z_{n}^{\nu}\right)-\psi_{n}^{\nu+1}\left(z_{n}^{\nu}-V\right)=-\alpha_{n}^{\nu}\left(z_{n}^{\nu}\right) \frac{\tau_{n+1}^{\nu}\left(z_{n}^{\nu}-V\right)}{\tau_{n+1}^{\nu+1}\left(z_{n}^{\nu}\right) \tau_{n}^{\nu+1}\left(z_{n}^{\nu}-V\right)} C,
$$


while the evaluation of $I_{n-1}^{\nu+1}$ at the divisor $\mathcal{T}_{n}^{\nu}$ implies

$$
\psi_{n}^{\nu+1}\left(z_{n}^{\nu}+V\right)-\psi_{n-1}^{\nu+1}\left(z_{n}^{\nu}\right)=\alpha_{n}^{\nu}\left(z_{n}^{\nu}\right) \frac{\tau_{n-1}^{\nu}\left(z_{n}^{\nu}+V\right)}{\tau_{n}^{\nu+1}\left(z_{n}^{\nu}+V\right) \tau_{n-1}^{\nu+1}\left(z_{n}^{\nu}\right)} C .
$$

The left-hand-side of the difference of (4.5) and (4.6) is the same as that of the difference of (4.7) of (4.8); equating the right-hand-sides of these differences yields (4.4).

Formulation $(A)$ of our main theorem implies that the assumption of lemma 4.1 is satisfied for $C=c_{3}, z \in \mathbb{C}^{g}$, and

$$
\tau_{n}^{\nu}(z)=\theta(U n+(1-\nu) W+z)\left(c_{1}^{(l, z)} c_{2}^{n}\right)^{\nu-\frac{1}{2}}
$$

where $l \in \mathbb{C}^{g}$ is a vector such that $(l, V)=1$. Then from (4.4) for $\nu=0$ we get on the divisor $\mathcal{T}_{0}^{0}$, i.e. for $\theta(Z)=\theta(z+W)=0$,

$$
\begin{array}{r}
\tau_{1}^{1}(z) \tau_{0}^{1}(z+V) \tau_{-1}^{0}(z-V)+\tau_{1}^{0}(z+V) \tau_{0}^{1}(z-V) \tau_{-1}^{1}(z) \\
=c_{3}^{2}\left(\tau_{1}^{1}(z) \tau_{0}^{1}(z-V) \tau_{-1}^{0}(z+V)+\tau_{1}^{0}(z-V) \tau_{0}^{1}(z+V) \tau_{-1}^{0}(z)\right),
\end{array}
$$

which upon substituting (4.9) yields, after canceling the common factors,

$$
\begin{array}{r}
c_{1}^{2} c_{2}^{2} \theta(Z+U-W) \theta(Z+V-W) \theta(Z-U-V) \\
+\theta(Z+U+V) \theta(Z-V-W) \theta(Z-U-W) \\
=c_{2}^{2} c_{3}^{2} \theta(Z+U-W) \theta(Z-V-W) \theta(Z+V-U) \\
+c_{1}^{2} c_{3}^{2} \theta(Z-V+U) \theta(Z+V-W) \theta(Z-U-W)
\end{array}
$$

which is identical to equation (1.6) with the minus sign chosen for $W$ (and correspondingly the constants $c_{1}$ and $c_{2}$ appearing in positive power). Similarly the case of $\nu=1, n=0$ of formula (4.4) yield the plus sign case of (1.6). The implication $(A) \Rightarrow(C)$ in the main theorem is thus proved.

Let us now show that $(C)$ can also be obtained as a corollary of a more general fourth order relation for Prym theta-functions. As it was mentioned above, in [7] it was proved that equation (1.1) implies the five-term equation (2.26). Note, that all the pairs of indices have sums of the same parity, i.e. equation (2.26) is in fact a pair of equations on two functions $\psi$ defined on two sublattices of the variables $(n, m)$.

The statement that $\psi_{n, m}$ satisfy (2.26) can be proved directly. Indeed all the functions involved in the equation are in

$$
H^{0}\left(D+(n+1) P_{1}^{+}-(n-1) P_{1}^{-}+(m+1) P_{2}^{+}-(m-1) P_{2}^{-}+\nu\left(P_{3}^{+}-P_{3}^{-}\right)\right)
$$

By the Riemann-Roch theorem the dimension of the latter space is 4 . Hence, any five elements of this space are linearly dependent, and it remains to find the coefficients of (2.26) 
by a comparison of singular terms at the points $P_{1}^{ \pm}, P_{2}^{ \pm}$. For $\left.n+m=0 \bmod 2\right)$ we get

$$
\begin{aligned}
\tilde{a}_{n, m} & =c_{1}^{2} c_{3}^{2} \frac{\theta\left(Z_{n, m}+V\right) \theta\left(Z_{n, m}+U-V+W\right)}{\theta\left(Z_{n, m}-V\right) \theta\left(Z_{n, m}+U+V+W\right)}, \\
\tilde{b}_{n, m} & =c_{2}^{2} c_{3}^{2} \frac{\theta\left(Z_{n, m}+U\right) \theta\left(Z_{n, m}-U+V+W\right)}{\theta\left(Z_{n, m}-U\right) \theta\left(Z_{n, m}+U+V+W\right)}, \\
\tilde{c}_{n m} & =c_{1}^{2} c_{2}^{2} \frac{\theta\left(Z_{n, m}+U\right) \theta\left(Z_{n, m}+V\right) \theta\left(Z_{n, m}-U-V+W\right)}{\theta\left(Z_{n, m}+U\right) \theta\left(Z_{n, m}-V\right) \theta\left(Z_{n, m}+U+V+W\right)},
\end{aligned}
$$

where $Z_{n, m}=Z+U n+V m$. From the normalization of $\psi_{n, m}$ it follows that

$$
\tilde{d}_{n m}=1-\tilde{a}_{n, m}-\tilde{b}_{n, m}+\tilde{c}_{n, m}
$$

Substituting here (2.23) and (4.12-4.13) proves the following statement.

Lemma 4.2 For any four points $A, U, V, W$ on the image $\Gamma \hookrightarrow \mathcal{P}(\Gamma)$, and any $Z \in \mathcal{P}(\Gamma)$ the following equation holds:

$$
\begin{aligned}
& \theta(Z+W) \times[\quad \theta(A+U+V+Z) \theta(Z-U) \theta(Z-V) \\
& -c_{1}^{2} c_{3}^{2} \theta(A+U-V+Z) \theta(Z-U) \theta(Z+V) \\
& -c_{2}^{2} c_{3}^{2} \theta(A-U+V+Z) \theta(Z+U) \theta(Z-V) \\
& \left.+c_{1}^{2} c_{2}^{2} \theta(A-U-V+Z) \theta(Z+U) \theta(Z+V)\right]= \\
& =\theta(A+Z) \times[\quad \theta(W+U+V+Z) \theta(Z-U) \theta(Z-V) \\
& -c_{1}^{2} c_{3}^{2} \theta(W+U-V+Z) \theta(Z-U) \theta(Z+V) \\
& -c_{2}^{2} c_{3}^{2} \theta(W-U+V+Z) \theta(Z+U) \theta(Z-V) \\
& \left.+c_{1}^{2} c_{2}^{2} \theta(W-U-V+Z) \theta(Z+U) \theta(Z+V)\right] .
\end{aligned}
$$

To the best of the authors' knowledge equation (4.14) is a new identity for Prym thetafunctions. For $Z$ such that $\theta(W+Z)=0$ it is equivalent to equation (1.6) with minus sign chosen. The second equation of the pair (1.6) can be obtained from (2.26) considered for the odd case, i.e. for $n+m=1 \bmod 2$.

Wave solutions. In section 2 we proved that if $\theta(Z)$ is the Prym theta function, then equation (1.1) with $u$ as in (1.2) has not just one solution $\psi$ of the form (1.4) but a family of them parameterized by points $A$ in the image $\Gamma \longmapsto \mathcal{P}(\Gamma)$ under the Abel-Prym map. Note, however, that formulation $(C)$ of the main theorem does not involve $A$. The first step in proving the "only if" part of $(C)$ (and thus also of $(\mathrm{A})$ and $(\mathrm{B})$, which imply $(\mathrm{C})$ ) is to introduce a spectral parameter in the problem, i.e. to show that equations (1.6) are sufficient 
for the existence of certain formal solutions of equations (4.1). These solutions are functions of the form

$$
\psi_{n}^{\nu}(z)=k^{n} C^{(l, z)} \phi_{n}^{\nu}(z, k)
$$

where $k^{-1}$ is a formal parameter (eventually to be identified with the local coordinate on the curve), $\phi_{n}^{\nu}(z, k)$ is a regular series in $k^{-1}$, i.e.

$$
\phi_{n}^{\nu}(z, k)=\sum_{s=0}^{\infty} \xi_{n, s}^{\nu}(z) k^{-s}
$$

and $l \in \mathbb{C}^{d}$ is such that $(l, V)=1$.

The ultimate goal of this section is to show that such solutions exist with $\xi_{n, s}^{\nu}$ being holomorphic functions of $z \in \mathbb{C}^{g}$, defined outside the divisor $\theta(z+U n+(1-\nu) W)=0^{1}$. As we shall see below, an obstruction for the existence of such solutions is the "bad locus"

$$
\Sigma:=\Sigma^{0} \cup \Sigma^{1}
$$

where $\Sigma^{\nu}$ is the $V$-invariant subvariety of the divisor $\Theta+(\nu-1) W$ that is not $U$-invariant, i.e.

$$
\Sigma^{\nu}:=\left\{\begin{array}{lll}
Z \in X: & \forall n \in \mathbb{Z} & \theta(Z+n V+(1-\nu) W)=0 ; \\
& \exists n \in \mathbb{Z} & \theta(Z+U+n V+(1-\nu) W) \neq 0
\end{array}\right\}
$$

We will prove in lemma 4.10 that the bad locus is empty, but until then we construct the wave solutions with the desired properties only along certain affine subspaces of $\mathbb{C}^{g}$; then we will patch these together. ${ }^{2}$

Notations. Denote $\pi: \mathbb{C}^{g} \rightarrow X=\mathbb{C}^{g} / \Lambda$ the universal cover map for $X$. Let $Y$ be the Zariski closure of the group $\langle\mathbb{Z} V\rangle \subset X$. As an abelian subvariety, it is generated by its irreducible component $Y^{0}$, containing 0 , and by the point $V_{0}$ of finite order in $X$, such that $V-V_{0} \in Y^{0}, N V_{0}=\lambda_{0} \in \Lambda$. Shifting $Y$ if needed, we may assume, without loss of generality, that 0 is not in the bad locus $\Sigma$. Since any subset of $Y$ that is invariant under the shift by $V$ is dense in $Y$, this implies that $Y \cap \Sigma=\emptyset$.

We denote $\mathcal{C}:=\pi^{-1}(Y)$. Then $\mathcal{C}$ is a union of its connected component passing through zero (which is a linear subspace $\mathbb{V} \cong \mathbb{C}^{d} \subset \mathbb{C}^{g}$ ) and shifts by a preimage of a vector of finite order, i.e. we have $\mathcal{C}=\cup_{r \in \mathbb{Z}}\left(\mathbb{V}+r V_{0}\right)$. Denoting then $\Lambda_{0}:=\Lambda \cap \mathcal{C}$ we have $Y=\mathcal{C} / \Lambda_{0}$, and we can also write $\Lambda_{0}=\widetilde{\Lambda}_{0}+\mathbb{Z} V_{0}$, where $\widetilde{\Lambda}_{0}:=\Lambda \cap \mathbb{V}$.

In what follows we assume that $\tau_{n}^{\nu}(z)$ are non-vanishing identically holomorphic functions of the variable $z \in \mathcal{C}$ having the following factors of automorphy with respect to $\Lambda_{0}$ :

$$
\tau_{n}^{\nu}(z+\lambda)=\tau_{n}^{\nu}(z) e^{\left(z, \alpha_{\lambda}\right)+n \beta_{\lambda}+w_{\lambda}^{\nu}}
$$

where $\alpha_{\lambda}, \beta_{\lambda}^{\nu}$ are independent of $n$, and we define for further use

$$
b_{\lambda}^{\nu}:=e^{\beta_{\lambda}+w_{\lambda}^{\nu}-w_{\lambda}^{\nu+1}} .
$$

This means that $u_{n}^{\nu}(z)$ given by (4.2) is a section of some degree zero line bundle on $Y$.

\footnotetext{
${ }^{1}$ In $[15,16,17]$ the corresponding solutions were called $\lambda$-periodic reflecting the normalization leading to their definition. The idea of that normalization goes back to [20].

${ }^{2}$ The locus $\Sigma$ is an analog of singular locus considered in [28]. The authors are grateful to Enrico Arbarello for an explanation of its crucial role, which helped them to focus on the heart of the problem.
} 
Proposition 4.3 Suppose equation (4.4) for $\tau_{n}^{\nu}(z)$ holds. Then equations (4.1) with potentials $u_{n}^{\nu}(z)$ given by (4.2) have wave solutions of the form (4.15) such that

(i) the coefficients $\xi_{n, s}^{\nu}(z)$ of the formal series $\phi_{n}^{\nu}(z, k)$ are meromorphic functions of the variable $z \in \mathcal{C}$ with a simple pole at the divisor $\mathcal{T}_{n}^{\nu}$,

$$
\xi_{n, s}^{\nu}(z)=\frac{\tau_{n, s}^{\nu+1}(z)}{\tau_{n}^{\nu}(z)},
$$

where $\tau_{n, s}^{\nu+1}(z)$ is a holomorphic function (the shift from $\nu$ to $\nu+1$ is only for notational ease to simplify further formulas), and

$$
\tau_{n, 0}^{\nu}(z)=\tau_{n-1}^{\nu}(z)
$$

(ii) Each of the individual terms in the power series expansion of $\phi$ have the following automorphy properties (note we are not yet making any claims regarding $\phi$ as a whole)

$$
b_{\lambda}^{\nu} \xi_{n, s}^{\nu}(z+\lambda)-\xi_{n, s}^{\nu}(z)=\sum_{i=1}^{s} B_{i, n-s+i}^{\lambda} \xi_{n, s-i}^{\nu}(z),
$$

for any $\lambda \in \Lambda_{0}$ (notice that the coefficients depend on $i$ and in a sort of diagonal way on $n$, but do not depend on $\nu$, which will be important for the future computations).

Proof. Writing down the equation for $\psi$ in terms of the power series expansions in $k^{-s}$, and equating coefficient of $k^{-s}$ to zero (i.e. substituting $(4.2,4.15,4.16)$ into (4.1)) yields

$$
C \xi_{n+1, s+1}^{\nu}(z+V)-u_{n}^{\nu}(z)\left(\xi_{n+1, s+1}^{\nu+1}(z)-C \xi_{n, s}^{\nu+1}(z+V)\right)+\xi_{n, s}^{\nu}(z)=0 .
$$

For $s=-1$ equation (4.23) is satisfied with $\tau_{n, 0}^{\nu}$ given by (4.21), i.e. with

$$
\xi_{n, 0}^{\nu}(z)=\frac{\tau_{n-1}^{\nu+1}(z)}{\tau_{n}^{\nu}(z)} .
$$

We will now prove the lemma by induction in $s$. Let us assume inductively that for $r \leq s-1$ the functions $\xi_{n, r}^{\nu}(z)$ are known, for all $n$ and $\nu$, and satisfy the quasi-periodicity condition (4.22) above - it is customary in the subject to call such solutions Bloch solutions or Bloch functions.

The idea of the proof of the inductive step is as follows. We write down the equation relating $\tau_{n+1, s+1}^{\nu}$ (we are using $n+1$ instead of $n$ solely for the ease of notations - recall that the inductive assumption is for all $n$ ) to the $\tau$ for smaller values of $s$ (which we know inductively to exist and be holomorphic). From this equation we then get an explicit formula for $\tau_{n+1, s+1}^{\nu}$ on the divisor $\mathcal{T}_{n}^{\nu}$, i.e. for $\tau_{n}^{\nu}(z)=0$. We also get an explicit formula for $\tau_{n+1, s+1}^{\nu}$ for $z$ such that $\tau_{n}^{\nu}(z+V)=0$, which after translating the argument gives another formula for $\tau_{n+1, s+1}^{\nu}$ on the divisor $\mathcal{T}_{n}^{\nu}$. Once we verify that the two resulting formulas agree (this is a hard computation using the step of the induction), it will follow that $\tau_{n+1, s+1}^{\nu}$ restricted to 
$\mathcal{T}_{n}^{\nu}$ is in fact holomorphic and thus can be extended from this divisor holomorphically to $\mathbb{C}^{d}$. We now give the details of this argument.

Writing down equation (4.23) in terms of $\tau$ 's for arbitrary $s$, and clearing denominators yields

$$
\begin{aligned}
& C \tau_{n+1, s+1}^{\nu+1}(z+V) \tau_{n}^{\nu}(z)-C \tau_{n}^{\nu+1}(z+V) \tau_{n+1, s+1}^{\nu}(z) \\
& -C^{2} \tau_{n, s}^{\nu}(z+V) \tau_{n+1}^{\nu+1}(z)
\end{aligned}
$$

These equations can be easily solved on the divisor $\mathcal{T}_{n}^{\nu}$. Indeed, if we take $z=z_{n}^{\nu} \in \mathcal{T}_{n}^{\nu}$ here, the first term will vanish, and we get the following formula

$$
C \tau_{n+1, s+1}^{\nu}\left(z_{n}^{\nu}\right)=\frac{\tau_{n, s}^{\nu+1}\left(z_{n}^{\nu}\right) \tau_{n+1}^{\nu}\left(z_{n}^{\nu}+V\right)-C^{2} \tau_{n, s}^{\nu}\left(z_{n}^{\nu}+V\right) \tau_{n+1}^{\nu+1}\left(z_{n}^{\nu}\right)}{\tau_{n}^{\nu+1}\left(z_{n}^{\nu}+V\right)}
$$

Alternatively, using equation (4.25) for $\nu+1$ instead of $\nu$ and setting $z=z_{n}^{\nu}-V$, for $z_{n}^{\nu} \in \mathcal{T}_{n}^{\nu}$ as above, we get

$$
C \tau_{n+1, s+1}^{\nu}\left(z_{n}^{\nu}\right)=\frac{\tau_{n, s}^{\nu+1}\left(z_{n}^{\nu}\right) \tau_{n+1}^{\nu}\left(z_{n}^{\nu}-V\right)-C^{2} \tau_{n, s}^{\nu}\left(z_{n}^{\nu}-V\right) \tau_{n+1}^{\nu+1}\left(z_{n}^{\nu}\right)}{\tau_{n}^{\nu+1}\left(z_{n}^{\nu}-V\right)}
$$

For $\tau_{n+1, s+1}^{\nu}$ to have a chance to exist, these two expressions have to agree.

Lemma 4.4 If the inductive assumption (and the conditions of the proposition, in particular formula (4.4)) is satisfied for $s$, then the two expressions above for the function $\tau_{n+1, s+1}^{\nu}(z)$ restricted to the divisor $\mathcal{T}_{n}^{\nu}$ are equal.

Proof. Equating the two expressions obtained for $\tau_{n+1, s+1}^{\nu}$ on $\mathcal{T}_{n}^{\nu}$, we see that what we need to prove is the following identity

$$
\begin{aligned}
& \tau_{n, s}^{\nu+1}\left(z_{n}^{\nu}\right) \tau_{n+1}^{\nu}\left(z_{n}^{\nu}-V\right) \tau_{n}^{\nu+1}\left(z_{n}^{\nu}+V\right)-C^{2} \tau_{n, s}^{\nu}\left(z_{n}^{\nu}-V\right) \tau_{n+1}^{\nu+1}\left(z_{n}^{\nu}\right) \tau_{n}^{\nu+1}\left(z_{n}^{\nu}+V\right) \\
= & \tau_{n, s}^{\nu+1}\left(z_{n}^{\nu}\right) \tau_{n+1}^{\nu}\left(z_{n}^{\nu}+V\right) \tau_{n}^{\nu+1}\left(z_{n}^{\nu}-V\right)-C^{2} \tau_{n, s}^{\nu}\left(z_{n}^{\nu}+V\right) \tau_{n+1}^{\nu+1}\left(z_{n}^{\nu}\right) \tau_{n}^{\nu+1}\left(z_{n}^{\nu}-V\right) .
\end{aligned}
$$

To prove that this is the case, we will use the inductive assumption for $n-1, s-1$, and equation (4.4). Indeed, for $n-1, s-1$ equation (4.25) reads

$$
\begin{array}{cc}
C \tau_{n, s}^{\nu+1}(z+V) \tau_{n-1}^{\nu}(z) & -C \tau_{n-1}^{\nu+1}(z+V) \tau_{n, s}^{\nu}(z) \\
-C^{2} \tau_{n-1, s-1}^{\nu}(z+V) \tau_{n}^{\nu+1}(z) & +\tau_{n}^{\nu}(z+V) \tau_{n-1, s-1}^{\nu+1}(z)=0
\end{array}
$$

By the inductive assumption we know that this is satisfied. If we now take $z=z_{n}^{\nu}-V$, i.e. set $\tau_{n}^{\nu}(z+V)=0$ here, we get

$$
C^{2} \tau_{n-1, s-1}^{\nu}\left(z_{n}^{\nu}\right)=\frac{C \tau_{n, s}^{\nu+1}\left(z_{n}^{\nu}\right) \tau_{n-1}^{\nu}\left(z_{n}^{\nu}-V\right)-C \tau_{n-1}^{\nu+1}\left(z_{n}^{\nu}\right) \tau_{n, s}^{\nu}\left(z_{n}^{\nu}-V\right)}{\tau_{n}^{\nu+1}\left(z_{n}^{\nu}-V\right)} .
$$


Similarly, if we instead take the equation with $\nu+1$ instead of $\nu$, and take $z=z_{n}^{\nu}$, we get

$$
\tau_{n-1, s-1}^{\nu}\left(z_{n}^{\nu}\right)=\frac{C \tau_{n-1}^{\nu}\left(z_{n}^{\nu}+V\right) \tau_{n, s}^{\nu+1}\left(z_{n}^{\nu}\right)-C \tau_{n, s}^{\nu}\left(z_{n}^{\nu}+V\right) \tau_{n-1}^{\nu+1}\left(z_{n}^{\nu}\right)}{\tau_{n}^{\nu+1}\left(z_{n}^{\nu}+V\right)}
$$

Since we inductively assumed the existence and uniqueness of $\tau_{n-1, s-1}^{\nu}$, these two expressions must agree, which is to say that we have the following identity

$$
\begin{array}{r}
\tau_{n, s}^{\nu+1}\left(z_{n}^{\nu}\right) \tau_{n-1}^{\nu}\left(z_{n}^{\nu}-V\right) \tau_{n}^{\nu+1}\left(z_{n}^{\nu}+V\right)-\tau_{n, s}^{\nu}\left(z_{n}^{\nu}-V\right) \tau_{n-1}^{\nu+1}\left(z_{n}^{\nu}\right) \tau_{n}^{\nu+1}\left(z_{n}^{\nu}+V\right) \\
=C^{2} \tau_{n, s}^{\nu+1}\left(z_{n}^{\nu}\right) \tau_{n-1}^{\nu}\left(z_{n}^{\nu}+V\right) \tau_{n}^{\nu+1}\left(z_{n}^{\nu}-V\right)-C^{2} \tau_{n, s}^{\nu}\left(z_{n}^{\nu}+V\right) \tau_{n-1}^{\nu+1}\left(z_{n}^{\nu}\right) \tau_{n}^{\nu+1}\left(z_{n}^{\nu}-V\right)
\end{array}
$$

Notice now how similar this known identity is to formula (4.28) that we need to prove. Indeed, the coefficient of $\tau_{n, s}^{\nu+1}\left(z_{n}^{\nu}\right)$ in $(4.28)$ is equal to

$$
\tau_{n+1}^{\nu}\left(z_{n}^{\nu}-V\right) \tau_{n}^{\nu+1}\left(z_{n}^{\nu}+V\right)-\tau_{n+1}^{\nu}\left(z_{n}^{\nu}+V\right) \tau_{n}^{\nu+1}\left(z_{n}^{\nu}-V\right)
$$

Now using formula (4.4), which we know holds for $\tau$, we see that this coefficient is equal to

$$
\left(\tau_{n}^{\nu+1}\left(z_{n}^{\nu}-V\right) \tau_{n-1}^{\nu}\left(z_{n}^{\nu}+V\right)-\tau_{n}^{\nu+1}\left(z_{n}^{\nu}+V\right) \tau_{n-1}^{\nu}\left(z_{n}^{\nu}-V\right)\right) \frac{\tau_{n+1}^{\nu+1}\left(z_{n}^{\nu}\right)}{\tau_{n-1}^{\nu+1}\left(z_{n}^{\nu}\right)}
$$

Substituting this expression into (4.28) is equivalent to the identity

$$
\begin{gathered}
\tau_{n, s}^{\nu+1}\left(z_{n}^{\nu}\right) \frac{\tau_{n+1}^{\nu+1}\left(z_{n}^{\nu}\right)}{\tau_{n-1}^{\nu+1}\left(z_{n}^{\nu}\right)}\left(\tau_{n}^{\nu+1}\left(z_{n}^{\nu}-V\right) \tau_{n-1}^{\nu}\left(z_{n}^{\nu}+V\right)-C^{2} \tau_{n}^{\nu+1}\left(z_{n}^{\nu}+V\right) \tau_{n-1}^{\nu}\left(z_{n}^{\nu}-V\right)\right) \\
=\tau_{n, s}^{\nu}\left(z_{n}^{\nu}-V\right) \tau_{n+1}^{\nu+1}\left(z_{n}^{\nu}\right) \tau_{n}^{\nu+1}\left(z_{n}^{\nu}+V\right)-C^{2} \tau_{n, s}^{\nu}\left(z_{n}^{\nu}+V\right) \tau_{n+1}^{\nu+1}\left(z_{n}^{\nu}\right) \tau_{n}^{\nu+1}\left(z_{n}^{\nu}-V\right) .
\end{gathered}
$$

Multiplying this identity by $\frac{\tau_{n-1}^{\nu+1}\left(z_{n}^{\nu}\right)}{\tau_{n+1}^{\nu+1}\left(z_{n}^{\nu}\right)}$ yields formula (4.29), which we inductively know to hold. Thus formula (4.28) holds, and the lemma is proven.

Lemma 4.5 The function $\tau_{n+1, s+1}^{\nu}\left(z_{n}^{\nu}\right)$ given by (4.26) and (4.27) can be extended to a holomorphic function on the entire divisor $\mathcal{T}_{n}^{\nu}$.

Proof. The expression (4.26) for $\tau_{n+1, s+1}^{\nu}\left(z_{n}^{\nu}\right)$ is certainly holomorphic when $\tau_{n}^{\nu+1}\left(z_{n}^{\nu}+V\right)$ is non-zero, i.e. is holomorphic outside of $\mathcal{T}_{n}^{\nu} \cap\left(\mathcal{T}_{n}^{\nu+1}-V\right)$. Similarly the expression for $\tau_{n+1, s+1}^{\nu}$ given by formula (4.27) is holomorphic away from $\mathcal{T}_{n}^{\nu} \cap\left(\mathcal{T}_{n}^{\nu+1}+V\right)$.

We have assumed that the closure of the abelian subgroup generated by $V$ is everywhere dense. Thus for any $z_{n}^{\nu} \in \mathcal{T}_{n}^{\nu}$ there must exist some $N \in \mathbb{N}$ such that $z_{n}^{\nu}+(N+1) V \notin \mathcal{T}_{n}^{\nu+1}$; let $N$ moreover be the minimal such $N$. From (4.26) it then follows that $\tau_{n+1, s+1}^{\nu}$ can be extended holomorphically to the point $z_{n}^{\nu}+N V$. However, by lemma 4.4 we know that the expressions (4.27) and (4.26) agree. Thus expression (4.27) must also be holomorphic 
at $z_{n}^{\nu}+N V$; since its denominator there vanishes, it means that the numerator must also vanish, i.e. we must have

$$
C \tau_{n, s}^{\nu+1}\left(z_{n}^{\nu}+N V\right) \tau_{n+1}^{\nu}\left(z_{n}^{\nu}+(N-1) V\right)-\tau_{n, s}^{\nu}\left(z_{n}^{\nu}+(N-1) V\right) \tau_{n+1}^{\nu+1}\left(z_{n}^{\nu}+N V\right)=0 .
$$

But this expression is equal to the numerator of (4.26) at $z_{n}^{\nu}+(N-1) V$; thus $\tau_{n+1, s+1}^{\nu}$ defined from (4.26) is also holomorphic at $z_{n}^{\nu}+(N-1) V$ (the numerator vanishes, and the vanishing order of the denominator is one, since we are talking exactly about points on its vanishing divisor). Thus unless $N=0$ we have a contradiction, since $N$ was chosen minimal. For $N=0$, however, $z_{n}^{\nu}+V \notin \mathcal{T}_{n}^{\nu+1}$, and thus (4.26) defines $\tau_{n+1, s+1}^{\nu}$ holomorphically at $z_{n}^{\nu}$.

Recall now that an analytic function on an analytic divisor in $\mathbb{C}^{d}$ has a holomorphic extension to all of $\mathbb{C}^{d}([27])$. Therefore, there exists a holomorphic function $\widetilde{\tau}_{n+1, s+1}^{\nu}(z)$ extending the function given on the divisor $\mathcal{T}_{n}^{\nu}$ by the r.h.s. of (4.26) (by the above lemma, it is holomorphic, and thus the extension is holomorphic). It is then natural to attempt to use the function $\widetilde{\xi}_{n+1, s+1}^{\nu}:=\widetilde{\tau}_{n+1, s+1}^{\nu+1} / \tau_{n+1}^{\nu}$ for the proposition, but this cannot be done immediately, as such an extension does not need to be quasi-periodic, nor is going to be a solution of equation (4.23). We will thus need to adjust this extension appropriately.

We start by determining the quasi-periodicity properties: indeed, for $z_{n}^{\nu+1} \in \mathcal{T}_{n}^{\nu+1}$, where we know that $\widetilde{\tau}_{n+1, s+1}^{\nu+1}$ is given by $(4.26)$, we have

$$
\widetilde{\xi}_{n+1, s+1}^{\nu}\left(z_{n}^{\nu+1}\right)=-C \xi_{n, s}^{\nu}\left(z_{n}^{\nu+1}+V\right)+\frac{\tau_{n, s}^{\nu}\left(z_{n}^{\nu+1}\right) \tau_{n+1}^{\nu+1}\left(z_{n}^{\nu+1}+V\right)}{\tau_{n}^{\nu}\left(z_{n}^{\nu+1}+V\right) \tau_{n+1}^{\nu}\left(z_{n}^{\nu+1}\right)},
$$

from which by using the quasi-periodicity of $\tau_{n}$ (4.18) and that of $\tau_{n, s}(4.22)$, it follows that

$$
\begin{aligned}
b_{\lambda}^{\nu} \widetilde{\xi}_{n+1, s+1}^{\nu}\left(z_{n}^{\nu+1}+\lambda\right) & =-C\left(\xi_{n, s}^{\nu}\left(z_{n}^{\nu+1}+V\right)-\sum_{i=1}^{s} B_{i, n-s+i}^{\nu} \xi_{n, s-i}^{\nu}\left(z_{n}^{\nu+1}+V\right)\right) \\
& +\frac{\left(\tau_{n, s}^{\nu}\left(z_{n}^{\nu+1}\right)+\sum_{i=1}^{s} B_{i, n-s+i}^{\lambda} \tau_{n, s-i}^{\nu}\left(z_{n}^{\nu+1}\right)\right) \tau_{n+1}^{\nu+1}\left(z_{n}^{\nu+1}+V\right)}{\tau_{n}^{\nu}\left(z_{n}^{\nu+1}+V\right) \tau_{n+1}^{\nu}\left(z_{n}^{\nu+1}\right)}
\end{aligned}
$$

since the $e^{\left(2 z+V, \alpha_{\lambda}\right)+(2 n+1) \beta_{\lambda}}$ factors for the second term of (4.32) coming from (4.18) cancel in the numerator and denominator, and the remaining $e^{2 \omega_{\lambda}^{\nu+1}-2 \omega_{\lambda}^{\nu}}$ cancels with $b_{\lambda}^{\nu} / b_{\lambda}^{\nu+1}$. We now note that the terms in the r.h.s. split in pairs similar to those in (4.32) and we can thus simplify this to get

$$
0=b_{\lambda}^{\nu} \widetilde{\xi}_{n+1, s+1}^{\nu}\left(z_{n}^{\nu+1}+\lambda\right)-\widetilde{\xi}_{n+1, s+1}^{\nu}\left(z_{n}^{\nu+1}\right)-\sum_{i=1}^{s} B_{i, n-s+i}^{\lambda} \xi_{n+1, s+1-i}^{\nu}\left(z_{n}^{\nu+1}\right)
$$

This says that the function on the right-hand-side here - denote it by $g_{n+1, s+1}^{\lambda, \nu}(z)$ - vanishes for $z=z_{n}^{\nu+1} \in \mathcal{T}_{n}^{\nu+1}$ and has a pole for $z \in \mathcal{T}_{n+1}^{\nu}$. Using formula (4.24) for $\xi_{n, 0}^{\nu}$, we can then write

$$
g_{n+1, s+1}^{\lambda, \nu}(z)=f_{n+1, s+1}^{\lambda, \nu}(z) \xi_{n+1,0}^{\nu}(z)
$$


where $f_{n+1, s+1}^{\lambda, \nu}(z)$ is now holomorphic, and satisfies the twisted homomorphism relations

$$
f_{n+1, s+1}^{\lambda+\mu, \nu}(z)=f_{n+1, s+1}^{\lambda, \nu}(z+\mu)+f_{n+1, s+1}^{\mu, \nu}(z)
$$

We only know the function $\widetilde{\xi}$ to have the desired quasi-periodicity on the divisor $\mathcal{T}_{n}^{\nu+1}$, and would now like to adjust it so that the corrected function would have computable quasiperiodicity for all $z$. To achieve this, we need to add to $\widetilde{\xi}$ a summand involving $f$.

Indeed, $f$ defines an element of the first cohomology group of $\Lambda_{0}$ with coefficients in the sheaf of holomorphic functions, $f \in H_{g r}^{1}\left(\Lambda_{0}, H^{0}\left(\mathbb{C}^{d}, \mathcal{O}\right)\right)$. The arguments identical to that in the proof of part (b) of Lemma 12 in [28] show that there must then exist a holomorphic function $h_{n+1, s+1}^{\nu}(z)$ such that

$$
f_{n+1, s+1}^{\lambda, \nu}(z)=h_{n+1, s+1}^{\nu}(z+\lambda)-h_{n+1, s+1}^{\nu}(z)+E_{n+1, s+1}^{\lambda, \nu},
$$

where $E_{n+1, s+1}^{\lambda, \nu}$ is a (z-independent!) constant. By using equation (4.35) we observe that $E$ depends on $\lambda$ linearly, i.e. that

$$
E_{n+1, s+1}^{\lambda+\mu, \nu}=E_{n+1, s+1}^{\lambda, \nu}+E_{n+1, s+1}^{\mu, \nu}
$$

We then define

$$
\zeta_{n+1, s+1}^{\nu}(z):=\widetilde{\xi}_{n+1, s+1}^{\nu}(z)-h_{n+1, s+1}^{\nu}(z) \xi_{n+1,0}^{\nu}(z)
$$

Using (4.24) and (4.18), we first compute

$$
\xi_{n+1,0}^{\nu}(z+\lambda)=\frac{\tau_{n}^{\nu+1}(z+\lambda)}{\tau_{n+1}^{\nu}(z+\lambda)}=e^{\omega_{\lambda}^{\nu+1}-\omega_{\lambda}^{\nu}-\beta_{\lambda}} \frac{\tau_{n}^{\nu+1}(z)}{\tau_{n+1}^{\nu}(z)}=\frac{\xi_{n+1,0}^{\nu}(z)}{b_{\lambda}^{\nu}}
$$

and then compute the quasi-periodicity

$$
\begin{gathered}
b_{\lambda}^{\nu} \zeta_{n+1, s+1}^{\nu}(z+\lambda)-\zeta_{n+1, s+1}^{\nu}(z)=\left(b_{\lambda}^{\nu} \widetilde{\xi}_{n+1, s+1}^{\nu}(z+\lambda)-\widetilde{\xi}_{n+1, s+1}^{\nu}(z)\right) \\
-b_{\lambda}^{\nu} h_{n+1, s+1}^{\lambda, \nu}(z+\lambda) \xi_{n+1,0}^{\nu}(z+\lambda)+h_{n+1, s+1}^{\lambda, \nu}(z) \xi_{n+1,0}^{\nu}(z) \\
=\left(g_{n+1, s+1}^{\lambda, \nu}(z)+\sum_{i=1}^{s} B_{i, n-s+i}^{\lambda} \xi_{n+1, s+1-i}^{\nu}(z)\right)+\left(E_{n+1, s+1}^{\lambda, \nu}-f_{n+1, s+1}^{\lambda, \nu}(z)\right) \xi_{n+1,0}^{\nu}(z) \\
=E_{n+1, s+1}^{\lambda, \nu} \xi_{n+1,0}^{\nu}(z)+\sum_{i=1}^{s} B_{i, n-s+i}^{\lambda} \xi_{n+1, s+1-i}^{\nu}(z) .
\end{gathered}
$$

Now we have constructed a function $\zeta$ having the correct quasi-periodicity properties (though the first coefficient depends on $\nu$, so we'll need to deal with this below) but we still cannot take it to be the function $\xi_{n+1, s+1}^{\nu}$ that we are trying to define, as it may not satisfy the equation (4.23). We thus define $R_{n+1, s+1}^{\nu}$ to be the "error" obtained by plugging $\zeta$ into (4.23):

$R_{n+1, s+1}^{\nu}(z) \xi_{n+1,0}^{\nu}(z+V):=C \zeta_{n+1, s+1}^{\nu}(z+V)-u_{n}^{\nu}(z)\left(\zeta_{n+1, s+1}^{\nu+1}(z)-C \xi_{n, s}^{\nu+1}(z+V)\right)+\xi_{n, s}^{\nu}(z)$. 
Notice that for this to make sense we need to assume that we have been doing all of the above computations simultaneously for $\nu$ and $\nu+1$, so that indeed both $\zeta$ 's above are defined at this point.

From the previous lemma we know that the r.h.s of this formula has no pole at $\mathcal{T}_{n}^{\nu}$ and vanishes at $\mathcal{T}_{n}^{\nu+1}-V$, and thus $R_{n+1, s+1}^{\nu}$ is a holomorphic function of $z$. We can use $(4.22,4.39)$ to compute the transformation properties of $R$ under a shift by a vector $\lambda \in \Lambda_{0}$. Indeed, using (4.18) to compute $b_{\lambda}^{\nu} u_{n}^{\nu}(z+\lambda)=u_{n}^{\nu}(z) b_{\lambda}^{\nu+1}$, and using (4.38) for the l.h.s., we get, shifting by $\lambda$ and multiplying by $b_{\lambda}^{\nu}$, and subtracting the original function,

$$
\begin{array}{r}
\left(R_{n+1, s+1}^{\nu}(z+\lambda)-R_{n+1, s+1}^{\nu}(z)\right) \xi_{n+1,0}^{\nu}(z+V) \\
=C E_{n+1, s+1}^{\lambda, \nu} \xi_{n+1,0}^{\nu}(z+V)+\sum_{i=1}^{s} B_{i, n-s+i}^{\lambda} \xi_{n+1, s+1-i}^{\nu}(z+V) \\
-u_{n}^{\nu}(z)\left(E_{n+1, s+1}^{\lambda, \nu+1} \xi_{n+1,0}^{\nu+1}(z)+\sum_{i=1}^{s} B_{i, n-s+i}^{\lambda} \xi_{n+1, s+1-i}^{\nu+1}(z)\right. \\
\left.-C \sum_{i=1}^{s} B_{i, n-s+i}^{\lambda} \xi_{n, s-i}^{\nu+1}(z+V)\right)+\sum_{i=1}^{s} B_{i, n-s+i}^{\lambda} \xi_{n, s-i}^{\nu}(z) .
\end{array}
$$

Now note that for each constant $B_{i, n-s+i}^{\lambda}$ in the above expression the function it multiplies is exactly the r.h.s. of (4.23) for $n$ and some $j \leq s$, and thus vanishes identically (this uses in a crucial way the fact that $B$ 's do not depend on $\nu$ ). Using the formulas $(4.2,4.24)$ for $u_{n}^{\nu}$ and $\xi_{n+1,0}$, we get

$$
R_{n+1, s+1}^{\nu}(z+\lambda)-R_{n+1, s+1}^{\nu}(z)=C\left(E_{n+1, s+1}^{\lambda, \nu}-E_{n+1, s+1}^{\lambda, \nu+1}\right) .
$$

Moreover, by (4.37) we know that the $E^{\prime}$ s are linear functions of $\lambda$, i.e. that

$$
E_{n+1, s+1}^{\lambda, \nu}-E_{n+1, s+1}^{\lambda, \nu+1}=2 \ell_{n+1, s+1}^{\nu}(\lambda)
$$

for some linear function $\ell$; note that $\ell_{n+1, s+1}^{\nu}(z)=-\ell_{n+1, s+1}^{\nu+1}(z)$. It then follows that the difference $R-2 \ell$ is periodic with respect to shifts by $\Lambda_{0}$, and is thus constant, i.e. we have then $R_{n+1, s+1}^{\nu}(z)=2 C \ell_{n+1, s+1}^{\nu}(z)+2 A^{\nu}$. We can now introduce one last correction and finally define

$$
\xi_{n+1, s+1}^{\nu}(z):=\zeta_{n+1, s+1}^{\nu}(z)-\left(\ell_{n+1, s+1}^{\nu}(z-V / 2)+A^{\nu}+l(z)\right) \xi_{n+1,0}^{\nu}(z),
$$

where $l(z)$ is a linear function such that $l(V)=A^{\nu}+A^{\nu+1}$. These functions are going to be solutions of (4.23); indeed, the new error term is equal to

$$
\begin{gathered}
R_{n+1, s+1}^{\nu}(z) \xi_{n+1,0}^{\nu}(z+V)-\left(\ell_{n+1, s+1}^{\nu}(z+V / 2)+A^{\nu}+l(z+V)\right) \xi_{n+1,0}^{\nu}(z+V) \\
+u_{n}^{\nu}(z)\left(\ell_{n+1, s+1}^{\nu+1}(z-V / 2)+A^{\nu+1}+l(z)\right) \xi_{n+1,0}^{\nu+1}(z) \\
=\xi_{n+1,0}^{\nu}(z+V)\left(R_{n+1, s+1}^{\nu}(z)-\ell_{n+1, s+1}^{\nu}(z+V / 2)-A^{\nu}-l(z+V)+\ell_{n+1, s+1}^{\nu+1}(z-V / 2)+A^{\nu+1}+l(z)\right)
\end{gathered}
$$




$$
=\xi_{n+1,0}^{\nu}(z+V)\left(2 \ell_{n+1, s+1}^{\nu}(z)+2 A^{\nu}-\ell_{n+1, s+1}^{\nu}(z)-A^{\nu}-l(V)-\ell_{n+1, s+1}^{\nu}(z)+A^{\nu+1}\right)=0,
$$

where we used the definitions (4.2),(4.21) and definitions of $\ell, l$, and $A$.

We now need to check that the functions $\xi$ satisfy the quasi-periodicity conditions (4.22). From $(4.38,4.39)$ it follows that

$$
\begin{gathered}
b_{\lambda}^{\nu} \xi_{n+1, s+1}^{\nu}(z+\lambda)-\xi_{n+1, s+1}^{\nu}(z)=\left(E_{n+1, s+1}^{\lambda, \nu}-\ell_{n+1, s+1}^{\nu}(\lambda)-l(\lambda)\right) \xi_{n+1,0}^{\nu}(z) \\
+\sum_{i=1}^{s} B_{i, n-s+i}^{\nu} \xi_{n+1, s+1-i}^{\nu}(z)
\end{gathered}
$$

which means that the function $\xi_{n+1, s+1}^{\nu}$ satisfies the quasi-periodicity condition (4.22), if we take

$$
B_{n+1, s+1}^{\lambda}:=E_{n+1, s+1}^{\lambda, \nu}-\ell_{n+1, s+1}^{\nu}(\lambda)-l(\lambda)=\frac{E_{n+1, s+1}^{\lambda, \nu}+E_{n+1, s+1}^{\lambda, \nu+1}}{2}-l(\lambda)
$$

(notice that this does not depend on $\nu$, as required in formula (4.22). Observe that the $B$ we construct is going to depend on the choice of the linear function $l(\lambda)$. We have thus constructed a quasi-periodic solution for $s+1$ and proved the inductive step of the proposition.

Corollary 4.6 For $\xi_{n, s}^{\nu}$ and $\xi_{n, s}^{\nu+1}$ fixed, the solutions of (4.23), for both $\nu$ and $\nu+1$, are unique up to the transformation

$$
\xi_{n+1, s+1}^{\nu}(z) \longmapsto \xi_{n+1, s+1}^{\nu}(z)+(c+l(z)) \xi_{n+1,0}^{\nu}(z)
$$

where $c$ is a constant, and $l$ is a linear function on $\mathcal{C}$ such that $l(V)=0$, both of them independent of $\nu$.

Proof. This follows by tracing the ambiguity of the choices involved in the proof of the above lemma. Alternatively, one can prove this directly by investigating the quasi-periodicity properties of the difference of two solutions of (4.23).

To fix the freedom of choosing $\xi_{n+1, s+1}^{\nu}$, we would now like to fix the quasi-periodicity condition to be the same for all $n$, and to be as simple as possible. Similarly to the case of a non-degenerate trisecant treated in [17], there may be a problem here in that the functions $\xi_{n, s}^{\nu}$ may turn out to be periodic (in our case by "periodic" we should mean $b_{\lambda_{j}} \xi_{n, s}^{\nu}\left(z+\lambda_{j}\right)=$ $\left.\xi_{n, s}^{\nu}(z)\right)$. Similarly to the situation in that paper, note that the space of periodic functions with a pole on the divisor $\mathcal{T}_{n}^{\nu}$ is the space of sections of some line bundle, and thus finitedimensional. Since all divisors $\mathcal{T}_{n}^{\nu}$ differ by shifts, there is an upper bound on this dimension independent of $n$ and $\nu$.

It then follows that the functions $\xi_{n, s}^{\nu}$, for $n$ fixed, and $s$ and $\nu$ varying, are linearly independent. Indeed, suppose that there were some linear relation among them, with the 
maximal value of $s$ involved in this relation being equal to $S$. But then solving equations (4.23) with $\nu$ and with $\nu+1$, allows one to express $\xi_{n, S}^{\nu}$ in terms of $\xi_{n-1, S-1}^{\nu}$ and $\xi_{n-1, S-1}^{\nu+1}$, and thus obtain a linear relation among the $\xi$ 's with index $n-1$, and maximal $s$ being equal to $S-1$. By downward induction, we can get to $S=0$ and get a contradiction with the fact that $\xi_{n, 0}^{\nu} \neq 0$ and is not proportional to $\xi_{n, 0}^{\nu+1}$. Note, moreover, that if for some $s$ the function $\xi_{n, s}^{\nu}$ is not periodic, this would mean that some $B$ is non-zero, and thus $\xi_{n, s+i}^{\nu}$ could not be periodic for any $i>0$, as the term in (4.22) with this non-zero $B$ would be linearly independent with all the other terms on the right-hand-side there.

Lemma 4.7 Let $\lambda_{0}, \lambda_{1}, \ldots, \lambda_{d}$ be a set of $\mathbb{C}$-linear independent vectors in $\Lambda_{0}$. Suppose equations (4.23) have periodic solutions for $i<r$ (and any $n$ and $\nu$ ), i.e. that there are some $\Xi_{n, i}^{\nu}(z)$ such that

$$
b_{\lambda_{j}}^{\nu} \Xi_{n, i}^{\nu}\left(z+\lambda_{j}\right)-\Xi_{n, i}^{\nu}(z)=0
$$

for all $i<r$, all $n$ and $\nu$, and such that $\Xi_{n, 0}^{\nu}=\xi_{n, 0}^{\nu}$ is given by (4.24). Suppose also that there are quasi-periodic solutions $\Xi_{n, r}^{\nu}$ with

$$
b_{\lambda_{j}}^{\nu} \Xi_{n, r}^{\nu}\left(z+\lambda_{j}\right)-\Xi_{n, r}^{\nu}(z)=A_{j} \xi_{n, 0}^{\nu}(z) \quad \forall j=0, \ldots, d,
$$

for all $n$, where $A_{j}$ are some constants such that there does not exist a linear form $l$ on $\mathcal{C}$ with $l\left(\lambda_{j}\right)=A_{j}$, and $l(V)=0$ (i.e. such that the scalar product of the vector $\vec{A}=\left(A_{1}, \ldots, A_{d}\right)$ and $V$ is non-zero). Then for all $s \geq r$, and all $n$ and $\nu$ equations (4.23) have quasi-periodic solutions satisfying (4.22) with $B_{i, n}^{\lambda_{j}}=A_{j} \delta_{i, r}$, i.e. there exist functions $\xi_{n, s}^{\nu}(z)$ for all $s \geq r$, all $n$ and $\nu$ such that

$$
b_{\lambda_{j}}^{\nu} \xi_{n, s}^{\nu}\left(z+\lambda_{j}\right)-\xi_{n, s}^{\nu}(z)=A_{j} \xi_{n, s-r}^{\nu}(z)
$$

(Note that we do not necessarily have $\xi_{n, i}^{\nu}(z)=\Xi_{n, i}^{\nu}(z)$ for $i \leq r$, but they satisfy the same quasi-periodicity, and solve the same equation (4.23).) Moreover, such $\xi_{n, s}^{\nu}(z)$ are unique up to adding $c_{n, s} \xi_{n, 0}^{\nu}(z)$, with $c_{n, s}$ being a constant dependent only on the remainder of $n$ modulo $r$.

Proof. We prove the lemma by induction in $s$, starting with $s=0$, with the inductive assumption being that functions $\Xi_{n, i}^{\nu}$ satisfying (4.23) and the quasiperiodicity condition (4.45) have been constructed for all $n$ and $\nu$, for all $i \leq r+s$, that they are "periodic" for $i<r$, and that moreover $\xi_{n, i}^{\nu}(z):=\Xi_{n, i}^{\nu}(z)$ for $i \leq s$ (so that the inductive assumption for $s=0$ is the assumption of the lemma).

From (4.43) we know that there must exist solutions $\widetilde{\xi}_{n, s+r+1}^{\nu}(z)$ of (4.23) for all $n$ and $\nu$, with quasi-periodicity

$$
b_{\lambda_{j}}^{\nu} \widetilde{\xi}_{n, s+r+1}^{\nu}\left(z+\lambda_{j}\right)=A_{j} \Xi_{n, s+1}^{\nu}(z)+B_{n, s+r+1}^{\lambda_{j}} \xi_{n, 0}^{\nu}(z),
$$

where $B$ are some new constants. The idea now is that we will adjust all the $\Xi_{n, s+i}^{\nu}$ for $0<i \leq r$ to another set of solutions of (4.23) with the same quasiperiodicity, so that $\Xi_{n, s+r+1}$ satisfying the quasi-periodicity condition (4.45) would exist. 
Indeed, suppose we take $\xi_{n+1, s+1}^{\nu}(z):=\Xi_{n+1, s+1}^{\nu}(z)+c_{n+1, s+1} \xi_{n+1,0}^{\nu}(z)$ for some constant $c_{n+1, s+1}$, independent of $\nu$ (if we added $l(z) \xi_{n+1,0}^{\nu}(z)$, the quasi-periodicity of $\xi_{n+1, s+1}^{\nu}(z)$ would no longer be the same as that of $\left.\Xi_{n+1, s+1}^{\nu}(z)\right)$. If we make such a change, we also need to add something (let's call it $f^{\nu}(z)$ ), to $\Xi_{n+2, s+2}^{\nu}(z)$, so that (4.23) is still satisfied. Since the $\Xi$ 's themselves satisfied (4.23), the corrections we introduce must also satisfy it, i.e. we must then have

$$
C f^{\nu}(z+V)-u_{n+1}^{\nu}(z)\left(f^{\nu+1}(z)-C c_{n+1, s+1} \xi_{n+1,0}^{\nu+1}(z+V)\right)+c_{n+1, s+1} \xi_{n+1,0}^{\nu}(z)=0,
$$

and the same for $\nu+1$. However, this is exactly the equation (4.23) that is satisfied by $c_{n+1} \Xi_{n+2,1}^{\nu}(z)$, and thus it follows that $f^{\nu}(z)=c_{n+1, s+1} \Xi_{n+2,1}^{\nu}(z)$ would work. Similarly we need to add $c_{n+1, s+1} \Xi_{n+i+1, i}^{\nu}(z)$ to each $\Xi_{n+i+1, s+i+1}^{\nu}(z)$, so that all of the equations (4.23) are satisfied. Finally in this way we will see that the necessary adjustment of $\widetilde{\xi}_{n+r+1, s+r+1}^{\nu}$ will be

$$
\Xi_{n+r+1, s+r+1}^{\nu}(z):=\widetilde{\xi}_{n+r+1, s+r+1}^{\nu}(z)+c_{n+1, s+1} \Xi_{n+r+1, r}^{\nu}(z)+l_{n+r+1}(z) \xi_{n+r+1,0}^{\nu}(z),
$$

where we will now need to allow the presence of a linear term to make the quasi-periodicity be (4.45) as desired. From (4.46) and (4.44) we can compute the quasi-periodicity to be

$$
\begin{gathered}
b_{\lambda_{j}}^{\nu} \Xi_{n+r+1, s+r+1}^{\nu}\left(z+\lambda_{j}\right)-\Xi_{n+r+1, s+r+1}^{\nu}(z) \\
=A_{j} \Xi_{n+r+1, s+1}^{\nu}(z)+\left(B_{n+r+1, s+r+1}^{\lambda_{j}}+c_{n+1, s+1} A_{j}+l_{n+r+1}\left(\lambda_{j}\right)\right) \xi_{n+r+1,0}^{\nu}(z) \\
=A_{j} \xi_{n+r+1, s+1}^{\nu}(z)+\left(B_{n+r+1, s+r+1}^{\lambda_{j}}+\left(c_{n+1, s+1}-c_{n+r+1, s+1}\right) A_{j}+l_{n+r+1}\left(\lambda_{j}\right)\right) \xi_{n+r+1,0}^{\nu}(z) .
\end{gathered}
$$

For this to be the desired property (4.45) we must have

$$
B_{n+r+1, s+r+1}^{\lambda_{j}}+\left(c_{n+1, s+1}-c_{n+r+1, s+1}\right) A_{j}+l_{n+r+1}\left(\lambda_{j}\right)=0 \quad \forall j=0, \ldots, d .
$$

For fixed $n$, this is a system of linear equations for the difference of the constants $c_{n+1, s+1}-$ $c_{n+r+1, s+1}$ and the coefficients of the linear form $l$. Recall that $l$ can be chosen arbitrary such that $l(V)=0$, i.e. if $\lambda_{0} \neq 0$ then the coefficients of $l$ span the $(d)$-dimensional space, in which by assumption $\vec{A}$ does not lie. Thus the rank of the matrix of coefficients is $d+1$, and this system of $d+1$ linear equations has a unique solution. If $\lambda_{0}=0$ then the dimension of linear forms $l$ is $d$, but periodicity condition for $\lambda_{0}$ is trivially satisfied. The inductive assumption is thus proven; note that as a result we are able to fix the differences $c_{n+1, s+1}-c_{n+r+1, s+1}$, and thus the constants only depend on the remainder of $n$ modulo $r$.

From local to global considerations. Until this point, we have only been working on $\mathcal{C}$, under the assumption that for all $n$ the functions $\tau_{n}^{\nu}(z)$ do not vanish identically. For $\tau_{n}^{\nu}$ given by (4.9) that is equivalent to the assumption that $U n \notin \Sigma$ for all $n$. We now observe that if a vector $Z \in \mathbb{C}^{g}$ is such that $Z+U n \notin \Sigma$ for all $n$, then by the same arguments we can construct wave solutions along the shifted affine subspaces $Z+\mathcal{C} \subset \mathbb{C}^{d}$. Since all the constructions are explicitly analytic, if we perturb $Z$ (while still staying away from $\Sigma-U n$ ), the solutions constructed along $Z+\mathcal{C}$ will change holomorphically with $Z$. Of course such 
solutions can only be constructed locally, while globally there may be a choice involved, and we may thus have a monodromy for this choice as we go around $\Sigma-U n$. Thus we cannot a priori expect $\xi_{n, s}(Z+z)$ (for $z \in \mathcal{C}, Z \in \mathbb{C}^{g}$ to be a global holomorphic function of $Z$.

Note that for fixed $n$ the functions $\xi_{n+1, s+1}^{\nu}(Z+z)$ exist if $Z+n U \notin \Sigma$, and $\xi_{n-i, s-i}^{\nu}(Z+z)$ exist for $0 \leq i \leq s$. Let us pass now from local to global setting. In this setting the recurrent equation (4.23) takes the form

$$
C \xi_{s+1}^{\nu}(Z+U+V)-u^{\nu}(Z)\left(\xi_{s+1}^{\nu+1}(Z+U)-C \xi_{s}^{\nu+1}(Z+V)\right)+\xi_{s}^{\nu}(Z)=0
$$

with

$$
u^{\nu}(Z)=C \frac{\tau^{\nu+1}(Z+U) \tau^{\nu+1}(Z+V)}{\tau^{\nu}(Z+U+V) \tau^{\nu}(Z)}
$$

where

$$
\tau^{\nu}(Z)=\theta(Z+(1-\nu) W)\left(c_{1}^{\left(l_{1}, Z\right)} c_{2}^{\left(l_{2}, Z\right)}\right)^{\nu-\frac{1}{2}}
$$

and $l_{1}, l_{2}$ are vectors such that $l_{1}(V)=l_{2}(U)=1, l_{1}(U)=l_{2}(V)=0$. In these notations the arguments in the proof of proposition 4.3 yield

Proposition 4.8 If equations (1.6) are satisfied, then:

(i) for $Z \notin \cup_{i=0}^{N}(\Sigma-i U)$ there exist functions $\tau_{s}^{\nu}(Z+z), 0 \leq s \leq N$, which are local holomorphic function of $Z$ and global holomorphic function of $z \in \mathcal{C}$, such that equations (4.47) hold for $\xi_{s}^{\nu}(Z)=\tau_{s}^{\nu+1}(Z) / \tau^{\nu}(Z)$, with $\tau_{0}^{\nu}(Z)=\tau^{\nu}(Z-U)$ (this is (4.24).

(ii) The functions $\xi_{s}$ satisfy the monodromy relations

$$
b_{\lambda}^{\nu} \xi_{s}^{\nu}(Z+z+\lambda)-\xi_{s}^{\nu}(Z+z)=\sum_{i=1}^{s} B_{i}^{\lambda}(Z) \xi_{s-i}^{\nu}(Z+z), \lambda \in \Lambda_{0}
$$

(iii) If $\xi_{s-1}$ is fixed then $\xi_{s}$ is unique up to the transformation

$$
\xi_{s}(z+Z) \longmapsto \xi_{s}(Z+z)+\left(c_{s}(Z)+l_{s}(Z, z)\right) \xi_{0},
$$

where $l_{s}(Z, z)$ is a linear form in $z$ such that $l_{s}(Z, V)=0$.

Lemma 4.9 Let $r$ be the minimal integer such that $\xi_{1}^{\nu}, \ldots, \xi_{r-1}^{\nu}$ are "periodic" functions of $z$ with respect to $\Lambda_{0}$, and such that there is no periodic solution $\xi_{r}^{\nu}$ of (4.47). Then the inductive assumptions of lemma (4.7) are satisfied, i.e. the quasi-periodicity coefficients $B_{i}^{\lambda}(Z)$ in (4.50) do not depend on $Z$ or $i-s$.

Proof. By assumption $\xi_{r-1}^{\nu}(z)$ is "periodic", i.e. we have

$$
0=b_{\lambda}^{\nu} \xi_{r-1}^{\nu}(Z+z+\lambda)-\xi_{r-1}^{\nu}(Z+z)=\sum_{i=1}^{r-1} B_{i}^{\lambda}(Z) \xi_{r-1-i}^{\nu}(Z+z)
$$


However, as it was noted above, the functions $\tau_{s}^{\nu}$, for $0 \leq s \leq r-1$ (recall that if not, by applying (4.47) we could produce a linear dependence having only one term, which is impossible), which means that all the coefficients $B_{i}^{\lambda}$ are zero for all $i \leq r-1$. Thus the monodromy of the next function is given by

$$
b_{\lambda}^{\nu} \xi_{r}^{\nu}(Z+z+\lambda)-\xi_{r}^{\nu}(Z+z)=B_{r}^{\lambda}(Z) \xi_{0}^{\nu}(Z+z)
$$

where we of course know $\xi_{0}^{\nu}$ explicitly, and $B_{r}^{\lambda}$ is a local function of $Z$ defined locally for

$$
Z \in X \backslash \bigcup_{i=0}^{r-1}(\Sigma-i V)
$$

From lemma 4.8 we know that the only ambiguity in the choice of the solutions $\xi_{n, r}^{\nu}(z)$ is given by (4.51). Recall that adding a linear function multiple will change the equation to be satisfied, while adding a constant multiple does not change the quasi-periodicity properties, so that finally $B_{r}^{\lambda}(Z)$ is independent of the ambiguity, and is well-defined as a holomorphic function of $Z \in X^{\prime}$. Since the locus $\Sigma \subset X$ is of codimension at least 2, by Hartogs theorem the function $B_{r}^{\lambda}(Z)$ can be extended holomorphically to all of $X$. Since $X$ is compact, this means that $B_{r}^{\lambda}(Z)$ is a constant, which we can denote $A_{\lambda}$ for the inductive assumption of lemma 4.7. If we had $\vec{A} \cdot V=0$, then by a transformation (4.43) with a suitable linear term we could get a new solution with $A_{\lambda_{i}}=0$ for $i=0 \ldots d$, i.e. the function $\xi_{n, r}^{\nu}(z)$ could be made "periodic", contradicting the way we chose $r$.

Lemma 4.10 In the setup of our construction the "bad locus" $\Sigma$ is actually empty, i.e. if equation (1.6) (part (C), the weakest assumption of our main theorem) is satisfied, then $\Sigma=\emptyset$.

Proof. The proof of this lemma is analogous to the proof of the similar statement for the fully discrete trisecant characterization of Jacobians treated in [17], once we first prove that $\Sigma^{0}=\Sigma^{1}$.

The only ambiguity in the definition of $\tau_{1}^{\nu}(Z)$ is in the choice of the coefficient $c_{1}$ in (4.51). Suppose there exists a point $A \in \Sigma^{0} \backslash \Sigma^{1}$, i.e. such that $\theta(A+N V) \neq 0=\theta(A+N V+W)$ for some $N$. Then locally near the point $A+N V$ choose some holomorphic branch of the function $\xi_{1}^{1}(Z)=\tau_{1}^{0}(Z) / \tau^{1}(Z)$. Doing this fixes the value of $c_{1}(Z)$ for all $Z$ near $A+N V$. However, since the ambiguity in the choice of $\xi_{1}^{0}(Z)=\tau_{1}^{1}(Z) / \tau^{0}(Z)$ is given by the same function $c_{1}(Z)$ (which did not depend on $\nu$ !) it means that for $Z$ in a neighborhood of $A+N V$, but away from $\Sigma^{0}$, we also have a fixed choice of $\xi_{1}^{0}(Z)$, and thus also of the holomorphic function $\tau_{1}^{1}(Z)$. Since $\Sigma^{0}$ has codimension at least 2 in $X$, the function $\tau_{1}^{1}(Z)$ can thus be extended to all points in a neighborhood of $A$, which is a contradiction. Thus we must have $\Sigma^{0} \subset \Sigma^{1}$, and of course by symmetry they are in fact equal.

We now prove in the same manner that $\Sigma=\Sigma+r U$ : above we used the fact that in (4.51) $c_{1}$ is independent of $\nu$, and now we will use $c_{1}(Z)=c_{1}(Z+r U)$. Indeed, suppose 
we have $A \in(\Sigma-r U) \backslash \Sigma$. This means that in a neighborhood of $p$ we can choose locally holomorphically the function $\tau_{1}^{\nu}(Z)$, i.e. chose a local holomorphic branch of $c_{1}(Z)$. However, since $c_{1}(Z)=c_{1}(Z+r U)$, this also fixes the choice of $c_{1}$ in a neighborhood of the point $p+r U$, and thus in a neighborhood of $p$, outside of $\Sigma-r U$, we have a holomorphic function $\tau_{1}^{\nu}(Z+r U)$, which now can be extended across $\Sigma-r U$, which we know to be of codimension at least two. This constructs a solution $\tau_{1}^{\nu}(A+r U)$, which contradicts the assumption $A \in(\Sigma-r U)$.

Since by definition $\Sigma$ has no subset invariant under a shift by $U$, this implies that either $\Sigma$ is empty, or $r>1$. Suppose now that $\Sigma$ is non-empty, so $r>1$. Recall that we have $\tau_{0}^{\nu}(Z)=\tau^{\nu}(Z-U)=\theta(Z+(1-\nu) W-U)$ const, and thus since $\Sigma^{0}=\Sigma^{1}$ we have $\left.\tau_{0}^{\nu}\right|_{\Sigma+U}=0$. Thus for any $Z \in \Sigma+U$, for $s=1$ the last two terms in (4.47) vanish, yielding

$$
C \xi_{1}^{\nu}(Z+U+V)-u^{\nu}(Z) \xi_{1}^{\nu+1}(Z+U)=0
$$

However, this is exactly equation (4.47) for $s=0$, which is solved by $\xi_{0}^{\nu}$, and thus all periodic with respect to $\Lambda_{0}$ solutions are constant multiples of $\xi_{0}^{\nu}$. By using (4.51) we can subtract this constant, and get a solution such that $\xi_{1}^{\nu}(Z+U)=0$ for any $Z \in \Sigma+U$, i.e. we have $\left.\xi_{1}^{\nu}\right|_{\Sigma+2 U}=0$.

Now we can repeat this process: indeed, for $s=2$ and $Z \in \Sigma+2 U$ the last two terms in (4.47) have $\xi_{1}^{\nu}(Z+V)$ and $\xi_{1}^{\nu}(Z)$ appearing, and thus vanish, so that as a result we see that $\xi_{2}^{\nu}$ on $\Sigma+3 U$ is a constant multiple of $\xi_{0}^{\nu}$. By using (4.51) again, we can make this multiple to be zero again. Repeating this a number of times, we will eventually get $\left.\xi_{r-1}^{\nu}\right|_{\Sigma+r U}=0$.

Since $\Sigma=\Sigma+r U$, we also have $\left.\tau^{\nu}\right|_{\Sigma+r U}=0$, and thus for $Z \in \Sigma+r U$ and $s=r-1$ the last two terms in (4.47) vanish to the second order - both factors of each summand vanish. Thus $\xi_{r}^{\nu}$ can be defined in a neighborhood of $\Sigma+r U=\Sigma$ as a holomorphic function vanishing on $\Sigma+r U$. However, this implies in particular that $b_{\lambda}^{\nu} \xi_{r}^{\nu}\left(Z+\lambda_{j}\right)-\xi_{r}^{\nu}(Z)=0$ for $Z \in \Sigma$ and any $\lambda_{j} \in \Lambda_{0}$, which contradicts the assumption that $\xi_{r}^{\nu}$ could not be periodic. The lemma is thus proven.

As shown above, if $\Sigma$ is empty, then the functions $\tau_{s}^{\nu}$ can be defined as global holomorphic functions of $Z \in \mathbb{C}^{g}$. Then, as a corollary of the previous lemmas we get the following statement.

Lemma 4.11 Suppose (1.6) for $\theta(Z)$ holds. Then there exists a pair of formal solutions

$$
\phi^{\nu}=\sum_{s=0}^{\infty} \xi_{s}^{\nu}(Z) k^{-s}
$$

of the equation

$$
k C \phi^{\nu}(Z+U+V, k)-u^{\nu}(Z)\left(k \phi^{\nu+1}(Z+U, k)-C \phi^{\nu+1}(Z+V, k)\right)-\phi^{\nu}(Z, k)=0,
$$

with $C=c_{3}$ and

$$
u^{\nu}(Z)=\frac{\tau^{\nu+1}(Z+U) \tau^{\nu+1}(Z+V)}{\tau^{\nu}(Z+U+V) \tau^{\nu}(Z)},
$$

where $\tau^{\nu}$ is given by (4.49), such that: 
(i) the coefficients $\xi_{s}^{\nu}$ of the formal series $\phi^{\nu}$ are of the form $\xi_{s}^{\nu}(Z)=\tau_{s}^{\nu+1}(Z) / \tau^{\nu}(Z)$, where $\tau_{s}^{\nu}(Z)$ are holomorphic functions;

(ii) $\phi^{\nu}(Z, k)$ is quasi-periodic with respect to the lattice $\Lambda$ and for the basis vectors $\lambda_{j}$ in $\mathcal{C}$ its monodromy relations have the form

$$
\phi^{\nu}\left(Z+\lambda_{j}\right)=\left(1+A_{\lambda_{j}} k^{-1}\right) \phi^{\nu}(Z, k), \quad j=1, \ldots, g,
$$

where $A_{\lambda_{j}}$ are constants such that there is no linear form on $\mathcal{C}$ vanishing at $V$, i.e. $l(V)=0$, and such that $l\left(\lambda_{j}\right)=A_{\lambda_{j}}$;

(iii) $\phi^{\nu}$ is unique up to the multiplication by a constant in $Z$ factor.

\section{The spectral curve}

In this section we finish a proof of the fact that condition $(C)$ of the main theorem characterizes Prym varieties. Indeed, in the previous section we showed that if $(C)$ holds, some quasi-periodic wave solutions can be constructed. In this section we show that these wave solutions are eigenfunctions of commuting difference operators, and identify $X$ with the Prym variety of the spectral curve of these operators. Much of the argument is analogous to that in $[16]$.

The formal series $\phi^{\nu}(Z, k)$ constructed in the previous section define a wave function

$$
\psi=\psi_{n m}(k):=k^{n} \phi^{\nu_{n m}}(n U+m V+Z, k) .
$$

This wave function determines a unique pseudo-difference operator $\mathcal{L}$ such that $\mathcal{L} \psi=k \psi$ (the coefficients of this $\mathcal{L}$ can be computed inductively term by term); we note that the ambiguity in the definition of $\phi^{\nu}(Z)$ (it is only defined up to a factor that is $T_{1}$-invariant) does not affect the coefficients of the wave operator. Therefore, its coefficients are of the form

$$
\mathcal{L}=\sum_{s=-1}^{\infty} w_{s}^{\nu_{n m}}(Z+n U+m V) T_{1}^{-s},
$$

where $w_{s}^{\nu}(Z)$ are well-defined meromorphic sections of line bundles on $X$ with automorphy properties given by (4.19).

As before, we define functions $\widetilde{F}_{j}$ by formula (3.63), i.e. we set

$$
\widetilde{F}_{j}:=\operatorname{res}_{T}\left(\left(\mathcal{L}^{j} T_{1}^{-1}-T_{1} \mathcal{L}^{j}\right)\left(T_{1}-T_{1}^{-1}\right)^{-1}\right)
$$

The definition of $\psi$ implies that these functions are of the form

$$
\widetilde{F}_{j}=\widetilde{F}_{j}^{\nu_{n m}}(U n+V m+Z)
$$

where $\widetilde{F}_{j}^{\nu}(Z)$ are meromorphic functions on $X$. 
Lemma 5.1 There exist vectors $V_{m}=\left\{V_{m, k}\right\} \in \mathbb{C}^{g}$ and constants $v_{m} \in \mathbb{C}$ such that

$$
\widetilde{F}_{j}^{\nu}(Z)=v_{j}+\frac{\partial}{\partial V_{j}}\left(\ln \tau^{\nu}(Z)-\ln \tau^{\nu+1}(Z+U)\right)
$$

Proof. Consider the formal series $\psi^{\sigma}$ given by (3.50). It has the form

$$
\psi_{n, m}^{\sigma}=k^{-n} \phi^{\sigma, \nu_{n, m}}(U n+V m+Z, k),
$$

where the coefficients of the formal series

$$
\phi^{\sigma, \nu}(Z, k)=\sum_{s=0}^{\infty} \xi_{s}^{\sigma, \nu}(Z) k^{-s}
$$

are difference polynomials in the coefficients of $\phi^{\nu}$ and $\phi^{\nu+1}$. Therefore, we know a priori that $\xi^{\sigma, \nu}(Z)$ are meromorphic functions, which may have poles for $Z \in \mathcal{T}^{\nu}, Z \in \mathcal{T}^{\nu+1}$, or for $Z$ on the translates of these two divisors by integer multiples of $U$. We claim that in fact these coefficients are of the form

$$
\xi_{s}^{\sigma, \nu}=\frac{\tau_{s}^{\sigma, \nu}(Z)}{\tau^{\nu}(Z)}
$$

where $\tau_{s}^{\sigma, \nu}(Z)$ are some holomorphic functions, i.e. that they only have simple poles at $\mathcal{T}^{\nu}$.

Indeed, we showed in section 3 that $\psi^{\sigma}$ solves the equation $H \psi^{\sigma}=0$. In section 4 we deduced from the statement $(C)$ of the main theorem the fact that $\psi^{\nu}$ may only have a simple pole on $\mathcal{T}^{\nu}$. By replacing $\psi^{\nu}$ by $\psi^{\sigma, \nu}$, and replacing $U$ by $-U$ we get from statement $(C)$ functional equations for $\tau_{s}^{\sigma, \nu}$ and in the same way deduce also that $\psi^{\sigma, \nu}$ only has pole at $\mathcal{T}^{\nu}$.

Equation (3.61) then implies that $\widetilde{\mathcal{F}}_{j}^{\nu}$ are the coefficients of the formal series

$$
-k+\left(k^{2}-1\right) \sum_{j=1}^{\infty} \widetilde{F}_{j}^{\nu}(Z)=k^{-1} \phi^{\sigma, \nu+1}(Z+U, k) \phi^{\nu}(Z, k)-k \phi^{\sigma, \nu}(Z, k) \phi^{\nu+1}(Z+U, k)
$$

It thus follows that $\widetilde{F}_{j}^{\nu}(Z)$ have simple poles only at the divisors $\mathcal{T}^{\nu}$ and $\mathcal{T}^{\nu+1}-U-$ these are the only possible poles of the right-hand-side. Moreover, equation (3.69) says (recall that $\mathbf{t}_{\mathbf{1}}$ is shifting the variable $n$, i.e. adding $U$ ) that there exist meromorphic functions $Q_{j}^{\nu}$ such that

$$
\widetilde{F}_{j}^{\nu}(Z)=Q_{j}^{\nu}(Z)-Q_{j}^{\nu+1}(Z+U)
$$

We know a priori that $Q_{j}^{\nu}$ may only have poles at $\mathcal{T}^{\nu}$ and $\mathcal{T}^{\nu+1}-U$. However, if there were a pole at $\mathcal{T}^{\nu+1}-U$, it would then mean that $Q_{j}^{\nu+1}(Z+U)$ would have a pole at $\mathcal{T}^{\nu}-2 U$, and since by our initial assumptions $U$ was not a point of order two, this is impossible. Thus $Q_{j}^{\nu}$ has simple pole only on $\mathcal{T}^{\nu}$, as desired for the expression (5.3) for $\widetilde{F}_{j}^{\nu}$ to be valid. The functions $\widetilde{F}_{j}^{\nu}$ are abelian functions. Therefore, the residue of $Q_{j}^{\nu}$ is a well-defined section of the theta-bundle restricted $\mathcal{T}^{\nu}$, i.e.

$$
\left.\left(Q_{j}^{\nu} \tau^{\nu}\right)\right|_{\mathcal{T}^{\nu}} \in H^{0}\left(\left.\tau^{\nu}\right|_{\mathcal{T}^{\nu}}\right)
$$


It is know that the later space is spanned by the directional derivatives of the theta function. Thus we see that there must exist some vector $V_{j}^{\nu} \in \mathbb{C}^{g}$ such that $Q_{j}^{\nu}-\left(\partial \ln \tau^{\nu}(Z) / \partial V_{j}^{\nu}\right)$ is a holomorphic function. The periodicity of $\widetilde{F}_{j}^{\nu}$ with respect to the lattice implies that $V_{j}^{\nu}=V_{j}^{\nu+1}$, and thus (5.3) holds.

Consider now the linear space spanned the functions $\left\{\widetilde{F}_{j}^{\nu}(Z), j=1, \ldots\right\}$. From (5.3) we see that there are only $g+1$ parameters involved in determining $\widetilde{F}_{j}^{\nu}$, and thus this space is at most $g+1$-dimensional. Therefore, for all but $\widetilde{g}:=\operatorname{dim}\left\{\widetilde{F}_{j}^{\nu}(Z)\right\}-1 \leq g$ positive integers $j$, there exist constants $c_{i, j}$ such that

$$
\widetilde{F}_{j}^{\nu}(Z)=c_{0, j}+\sum_{i=1}^{j-1} c_{i, j} \widetilde{F}_{i}^{\nu}(Z) .
$$

Let $I$ denote the subset of integers $j$ for which there are no such constants. We call this subset the gap sequence — the corresponding $\widetilde{F}_{j}^{\nu}$ form a basis.

Lemma 5.2 Let $\mathcal{L}$ be the pseudo-difference operator corresponding to the quasi-periodic (Bloch) wave function $\psi$ constructed above. Then, for the difference operators

$$
\widehat{L}_{j}:=L_{j}+\sum_{i=1}^{j-1} c_{i, j} L_{n-i}=0, \quad \forall j \notin I,
$$

the following equations are satisfied with some constants $a_{s, j}$ :

$$
\widehat{L}_{j} \psi=a_{j}(k) \psi, \quad a_{j}(k)=k^{j}+\sum_{s=1}^{\infty} a_{s, j} k^{j-s}
$$

Proof. From the proof of Theorem 3.5 we get

$$
\left[L_{j}, H\right] \equiv\left(\mathbf{t}_{2} \widetilde{F}_{j}-\widetilde{F}_{j}\right)\left(T_{1}-T_{2}\right) \bmod \mathcal{O}_{H}
$$

Therefore, operators $\widehat{L}_{j}$ and $H$ commute in $\mathcal{O} / \mathcal{O}_{H}$. Hence, if $\psi$ is a Bloch wave solution of (4.1), i.e. $H \psi=0$, then $\widehat{L}_{j} \psi$ is also a Bloch solution of the same equation. Since (4.1) has a unique solution up to multiplication by constant (i.e. the kernel of $H$ is one-dimensional), we must have $\widehat{L}_{j} \psi=a_{j}(Z, k) \psi$, where $a_{j}$ is $T_{1}$-invariant, i.e. $a_{j}(Z, k)=a_{j}(Z+U, k)$.

Note that the constant factor ambiguity in the definition of $\psi$ does not affect $a_{j}$, and thus $a_{j}$ are well-defined global meromorphic functions on $\mathbb{C}^{g} \backslash \Sigma$. Since the closure of $\mathbb{Z} U$ is dense in $X$, the $T_{1}$ invariance of $a_{j}$ implies that $a_{j}$ is a holomorphic function of $Z \in X$, and thus it is constant in $Z$ (note that we in fact have $a_{s, n}=-c_{s, n}$ for $s \leq n$ ).

If we set now $m=0$, the operator $\widehat{L}_{j}$ can be regarded as a $Z$-parametric family of ordinary difference operators $\widehat{L}_{j}^{Z}$. 
Corollary 5.3 The operators $\widehat{L}_{j}^{Z}$ commute with each other,

$$
\left[\widehat{L}_{i}^{Z}, \widehat{L}_{j}^{Z}\right]=0
$$

A theory of commuting difference operators containing a pair of operators of co-prime orders was developed in $[24,14]$. It is analogous to the theory of rank 1 commuting differential operators $[3,4,12,13,24]$ (relatively recently this theory was generalized to the case of commuting difference operators of arbitrary rank in [19].)

Lemma 5.4 Let $\mathcal{A}^{Z}$ be the commutative ring of ordinary difference operators spanned by the operators $\widehat{L}_{j}^{Z}$. Then there exists an irreducible algebraic curve $\Gamma$ of arithmetic genus $\hat{g}$ with involution $\sigma: \Gamma \longmapsto \Gamma$ such that for a generic $Z$ the ring $\mathcal{A}^{Z}$ is isomorphic to the ring of meromorphic functions on $\Gamma$ with the only poles at two smooth points $P_{1}^{ \pm}$, which are odd with respect to the involution $\sigma$. The correspondence $Z \rightarrow \mathcal{A}^{Z}$ defines a holomorphic map of $X$ to the space of odd torsion-free rank one sheaves $\mathcal{F}$ on $\Gamma$

$$
j: X \longmapsto \overline{\operatorname{Prym}}(\Gamma)=\operatorname{Ker}(1+\sigma) \subset \overline{\operatorname{Pic}}(\Gamma) .
$$

Proof. As shown in $[24,14]$ there is a natural correspondence

$$
\mathcal{A} \longleftrightarrow\left\{\Gamma, P_{ \pm}, \mathcal{F}\right\}
$$

between commutative rings $\mathcal{A}$ of ordinary linear difference operators containing a pair of monic operators of co-prime orders, and sets of algebro-geometric data $\left\{\Gamma, P_{ \pm},\left[k^{-1}\right]_{ \pm}, \mathcal{F}\right\}$, where $\Gamma$ is an algebraic curve with fixed first jets $\left[k^{-1}\right]_{ \pm}$of local coordinates $k_{ \pm}^{-1}$ in the neighborhoods of smooth points $P_{1}^{ \pm} \in \Gamma$, and $\mathcal{F}$ is a torsion-free rank 1 sheaf on $\Gamma$ such that

$$
h^{0}(\Gamma, \mathcal{F})=h^{1}\left(\Gamma, \mathcal{F}\left(n P_{+}-n P_{-}\right)=0 .\right.
$$

The correspondence becomes one-to-one if the rings $\mathcal{A}$ are considered modulo conjugation $\mathcal{A}^{\prime}=g \mathcal{A} g^{-1}$.

The construction of the correspondence (5.14) depends on a choice of initial point $n_{0}=0$. The spectral curve and the sheaf $\mathcal{F}$ are defined by the evaluations of the coefficients of generators of $\mathcal{A}$ at a finite number of points of the form $n_{0}+n$. In fact, the spectral curve is independent on the choice of $x_{0}$, but the sheaf does depend on it, i.e. $\mathcal{F}$ depends on the choice of $n_{0}$.

Using the shift of the initial point it is easy to show that the correspondence (5.14) extends to the commutative rings of operators whose coefficients are meromorphic functions of $x$. The rings of operators having poles at $n=0$ correspond to sheaves for which the condition (5.15) for $n=0$ is violated.

A commutative ring $\mathcal{A}$ of linear ordinary difference operators is called maximal if it is not contained in any larger commutative ring. The algebraic curve $\Gamma$ corresponding to a maximal ring is called the spectral curve of $\mathcal{A}$. The $\operatorname{ring} \mathcal{A}$ is isomorphic to the ring 
$A\left(\Gamma, P_{1}^{ \pm}\right)$of meromorphic functions on $\Gamma$ with the only pole at $P_{1}^{+}$, and vanishing at $P_{1}^{-}$. The isomorphism is given by the equation

$$
L_{a} \psi=a \psi, \quad L_{a} \in \mathcal{A}, a \in A\left(\Gamma, P_{1}^{ \pm}\right),
$$

where $\psi$ is a common eigenfunction of the commuting operators.

Let $\Gamma^{Z}$ be the spectral curve corresponding to the maximal ring $\hat{\mathcal{A}}^{Z}$ containing $\mathcal{A}^{Z}$. The eigenvalues $a_{j}(k)$ of the operators $\hat{L}_{j}^{Z}$ defined in (5.11) coincide with the Laurent expansions at $P_{1}^{+}$of the meromorphic functions $a_{j} \in A\left(\Gamma^{Z}, P_{ \pm}\right)$, and thus are $Z$-independent. Hence, the spectral curve $\Gamma^{Z}$ in fact does not depend on $Z$.

The functions $\psi^{\sigma}$ are eigenfunctions of $\widehat{L}_{j}$ :

$$
\widehat{L}_{j} \psi^{\sigma}=-a_{j}(k) \psi^{\sigma} .
$$

Hence, the correspondence $\psi \leftrightarrow \psi^{\sigma}$ gives rise to an involution $\sigma$ of the spectral curve. The eigenvalues $a_{j}$ are odd with respect to the involution, and thus the lemma is proved.

The next step is to consider deformations of $\mathcal{A}^{Z}$ defined by the discrete Novikov-Veselov hierarchy introduced in Section 3. Through this hierarchy we identified the space spanned by functions $\widetilde{F}_{j}$ with the tangent space to the orbit of the hierarchy. Lemma 5.1 identifies the orbit of the hierarchy with $Z+Y$, where $\mathrm{Y}$ is the closure of the group spanned by vectors $V_{j}$. The orbit of the NV hierarchy is the odd part of the orbit of two KadomtsevPetviashvili flows corresponding to points $P_{1}^{ \pm}$. It follows from [28] that the orbit of the discrete NV hierarchy is isomorphic to $\mathcal{P}(\Gamma)$. For a generic $Z$ the $\operatorname{ring} A^{Z}$ is a maximal odd ring. Therefore, we get

Lemma 5.5 For $Z \in \mathbb{C}^{g}$ generic, the orbit of $\mathcal{A}^{Z}$ under the $N V$ flows defines an isomorphism:

$$
i_{Z}: \mathcal{P}(\Gamma) \longmapsto Z+Y \subset X
$$

Corollary 5.6 The Prym variety $\mathcal{P}(\Gamma)$ of the spectral curve $\Gamma$ is compact.

The compactness of the Prym variety is not as restrictive as the compactness of the Jacobian (see [6]). Nevertheless, it implies an explicit description of the singular points of the spectral curve. The following result is due to Robert Friedman (see the appendix of [16]):

Corollary 5.7 The spectral curve $\Gamma$ is smooth outside of fixed points $q_{k}$ of the involution $\sigma$. The branches of $\Gamma$ at $q_{k}$ are linear and are not permuted by $\sigma$.

The arguments identical to that used at the end of [16] prove that in fact the singular points $q_{k}$ are at most double points. For a curve with at most double singular points all the sheafs $\mathcal{F}$ are line bundles. Therefore, the map $j$ in (5.13) is inverse to $i_{Z}$ in (5.18), and the main theorem is thus proven.

Acknowledgments. We would like to thank Enrico Arbarello, Robert Friedman and Takahiro Shiota, in very useful discussions with whom we have learned many things about the geometry of Prym varieties and Prym theta divisors. 


\section{References}

[1] E. Arbarello, I. Krichever, G. Marini, Characterizing Jacobians via flexes of the Kummer Variety, Math. Res. Lett. 13 (2006) 1, 109-123.

[2] A. Beauville, O. Debarre, Sur le problème de Schottky pour les variétés de Prym. Ann. Scuola Norm. Sup. Pisa Cl. Sci. 14 (1987) 4, 613-623 (1988).

[3] J.L.Burchnall, T.W. Chaundy, Commutative ordinary differential operators.I, Proc. London Math. Soc. 21 (1922), 420-440.

[4] J.L.Burchnall, T.W. Chaundy, Commutative ordinary differential operators. II, Proc. Royal Soc. London 118 (1928), 557-583.

[5] O. Debarre: Vers une stratification de l'espace des modules des variétés abéliennes principalement polarisées. Complex algebraic varieties (Bayreuth, 1990), 71-86, Lecture Notes in Math., 1507, Springer, Berlin, 1992.

[6] P. Deligne, D. Mumford, The irreducibility of the space of curves of given genus. Inst. Hautes Etudes Sci. Publ. Math. 36 (1969), 75-109.

[7] A. Doliwa, P. Grinevich, M. Nieszporski, P. M. Santini, Integrable lattices and their sub-lattices: from the discrete Moutard (discrete Cauchy-Riemann) 4-point equation to the self-adjoint 5-point scheme, arXiv:nlin/0410046.

[8] B.A. Dubrovin, I.M. Krichever, S.P. Novikov Schrödinger equation in magnetic field and Riemann surfaces, Dokl. Akad. Nauk SSSR 229 (1976), 1, 15-18.

[9] J.D. Fay, Theta functions on Riemann surfaces. Lecture Notes in Mathematics, Vol. 352. Springer-Verlag, Berlin-New York, 1973.

[10] J.D. Fay, On the even-order vanishing of Jacobian theta functions. Duke Math. J. 51 (1984) 1, 109-132.

[11] R. Gunning, Some curves in abelian varieties, Invent. Math. 66 (1982) 3, 377-389.

[12] I.M. Krichever, Integration of non-linear equations by methods of algebraic geometry, Funct. Anal. Appl. 11 (1977) 1, 12-26.

[13] I.M. Krichever, Methods of algebraic geometry in the theory of non-linear equations, Russian Math. Surveys 32 (1977) 6, 185-213.

[14] I.Krichever, Algebraic curves and non-linear difference equation, Uspekhi Mat. Nauk 33 (1978) 4, 215-216.

[15] I.Krichever, Integrable linear equations and the Riemann-Schottky problem, Algebraic geometry and number theory, 497-514, Progr. Math., 253, Birkhäuser Boston, Boston, MA, 2006. 
[16] I.Krichever, A characterization of Prym varieties. Int. Math. Res. Not. 2006, Art. ID 81476, 36 pp.

[17] I. Krichever, Characterizing Jacobians via trisecants of the Kummer Variety, math.AG/0605625.

[18] I. Krichever, Two-dimensional periodic difference operators and algebraic geometry. (Russian) Dokl. Akad. Nauk SSSR 285 (1985) 1, 31-36.

[19] I. Krichever, S. Novikov, Two-dimensional Toda lattice, commuting difference operators and holomorphic vector bundles, Uspekhi Mat. Nauk , 58 (2003) 3, 51-88.

[20] I.Krichever, D.H.Phong, On the integrable geometry of soliton equations and $N=2$ supersymmetric gauge theories. J. Differential Geom. 45 (1997) 2, 349-389.

[21] I. Krichever, P. Wiegman, A. Zabrodin, Elliptic solutions to difference non-linear equations and related many-body problems. Comm. Math. Phys. 193 (1998) 2, 373-396.

[22] D. Mumford Theta characteristics of an algebraic curve. Ann. Sci. École Norm. Sup. 4 (1971), 181-192.

[23] D. Mumford, Prym varieties. I. Contributions to analysis (a collection of papers dedicated to Lipman Bers), 325-350. Academic Press, New York, 1974.

[24] D. Mumford, An algebro-geometric construction of commuting operators and of solutions to the Toda lattice equation, Korteweg-de Vries equation and related non-linear equations. Proceedings Int.Symp. Algebraic Geometry, Kyoto, 1977, 115-153, Kinokuniya Book Store, Kyoto, 1978.

[25] S. Novikov, A. Veselov, Finite-gap two-dimensional periodic Schrödinger operators: exact formulae and evolution equations, Dokl. Akad. Nauk SSSR 279 (1984) 1, 20-24.

[26] G.Segal, G.Wilson, Loop groups and equations of KdV type, Inst. Hautes Etudes Sci. Publ. Math. 61 (1985), 5-65.

[27] J-P. Serre, Faisceaux algébriques cohérents, Ann. of Math. 61 (1955), 197-278.

[28] T. Shiota, Characterization of Jacobian varieties in terms of soliton equations, Invent. Math. 83 (1986) 2, 333-382.

[29] G.E. Welters, A criterion for Jacobi varieties, Ann. of Math., 120 (1984) 3, 497-504. 